\title{
High-resolution X-Ray Spectroscopy of the Seyfert 1 Galaxy Mrk 1040. Revealing the Failed Nuclear Wind with Chandra
}

\author{
J. N. Reeves ${ }^{1,2}$, V. Braito ${ }^{1,3}$, E. Behar ${ }^{4,5}$, T. C. Fischer ${ }^{6}$, S. B. Kraemer ${ }^{7}$, A. Lobban ${ }^{2,8}$, E. Nardini ${ }^{2,9}$, D. Porquet ${ }^{10}$, and \\ T. J. Turner ${ }^{11}$ \\ ${ }^{1}$ Center for Space Science and Technology, University of Maryland Baltimore County, 1000 Hilltop Circle, Baltimore, MD 21250, USA; jreeves@ umbc.edu \\ ${ }^{2}$ Astrophysics Group, School of Physical and Geographical Sciences, Keele University, Keele, Staffordshire, ST5 5BG, UK; j.n.reeves@keele.ac.uk \\ ${ }^{3}$ INAF-Osservatorio Astronomico di Brera, Via Bianchi 46 I-23807 Merate (LC), Italy \\ ${ }^{4}$ Department of Physics, Technion, Haifa 32000, Israel \\ ${ }^{5}$ Department of Astronomy, University of Maryland, College Park, MD 20742, USA \\ ${ }^{6}$ Astrophysics Science Division, NASA Goddard Space Flight Center, Code 665, Greenbelt, MD 20771, USA \\ ${ }^{7}$ Institute for Astrophysics and Computational Sciences, Department of Physics, The Catholic University of America, Washington, DC 20064, USA \\ ${ }^{8}$ Department of Physics and Astronomy, University of Leicester, University Road, Leicester LE1 7RH, UK \\ ${ }^{9}$ INAF-Arcetri Astrophysical Observatory, Largo Enrico Fermi 5, I-50125 Firenze, Italy \\ ${ }^{10}$ Université de Strasbourg, CNRS, Observatoire astronomique de Strasbourg, UMR 7550, F-67000 Strasbourg, France \\ ${ }^{11}$ Department of Physics, University of Maryland Baltimore County, 1000 Hilltop Circle, Baltimore, MD 21250, USA \\ Received 2016 December 21; revised 2017 January 27; accepted 2017 January 29; published 2017 February 28
}

\begin{abstract}
High-resolution X-ray spectroscopy of the warm absorber in the nearby X-ray bright Seyfert 1 galaxy Mrk 1040 is presented. The observations were carried out in the 2013-2014 timeframe using the Chandra High Energy Transmission Grating with a total exposure of $200 \mathrm{ks}$. A multitude of absorption lines from $\mathrm{Ne}, \mathrm{Mg}$, and $\mathrm{Si}$ are detected from a wide variety of ionization states. In particular, the detection of inner K-shell absorption lines from $\mathrm{Ne}, \mathrm{Mg}$, and $\mathrm{Si}$, from charge states ranging from F-like to Li-like ions, suggests the presence of a substantial amount of low-ionization absorbing gas, illuminated by a steep soft X-ray continuum. The observations reveal at least three warm absorbing components ranging in ionization parameter from $\log \left(\xi / \mathrm{erg} \mathrm{cm} \mathrm{s}^{-1}\right)=0-2$ and with column densities of $N_{\mathrm{H}}=1.5-4.0 \times 10^{21} \mathrm{~cm}^{-2}$. The velocity profiles imply that the outflow velocities of the absorbing gas are low and within $\pm 100 \mathrm{~km} \mathrm{~s}^{-1}$ of the systemic velocity of Mrk 1040, which suggests that any outflowing gas may have stalled in this AGN on large enough scales. The warm absorber is likely located far from the black hole, within $300 \mathrm{pc}$ of the nucleus, and is spatially coincident with emission from an extended narrowline region as seen in the Hubble Space Telescope images. The iron K-band spectrum reveals only narrow emission lines, with $\mathrm{Fe} \mathrm{K} \alpha$ at $6.4 \mathrm{keV}$ consistent with originating from reflection off Compton-thick pc-scale reprocessing gas.
\end{abstract}

Key words: galaxies: active - galaxies: individual (Mrk 1040) - X-rays: galaxies

\section{Introduction}

Material existing within the vicinity of the supermassive black hole in an active galactic nucleus (AGN) can significantly modify the resulting X-ray spectrum. Consequently, detailed studies of the reprocessed primary X-ray emission can reveal valuable information about the kinematics and geometry of the central nucleus. In the soft X-ray band (below $2 \mathrm{keV}$ ), the dominant reprocessing mechanism is absorption by photoionized (or "warm") material along the line of sight. In fact, systematic X-ray studies of AGN with ASCA, Chandra, XMMNewton, and Suzaku have established that at least half of all Type 1 Seyferts host warm absorbers (Reynolds 1997; George et al. 1998; Crenshaw et al. 2003; Porquet et al. 2004; Blustin et al. 2005; McKernan et al. 2007; Tombesi et al. 2013). When observed at high spectral resolution, for instance with the X-ray grating spectrometers onboard either Chandra or XMM-Newton, the warm absorber is seen to give rise to numerous narrow absorption lines from astrophysically abundant elements such as $\mathrm{C}, \mathrm{N}, \mathrm{O}, \mathrm{Ne}, \mathrm{Mg}, \mathrm{Si}, \mathrm{S}$, and $\mathrm{Fe}$ (Kaastra et al. 2000; Blustin et al. 2002; Kaspi et al. 2002; McKernan et al. 2003). The warm absorption is often seen to be outflowing and can arise from gas covering a wide (several orders of magnitude) range of column densities and ionization parameters.

Indeed, the X-ray spectral signatures of the warm absorber range can arise from very low ionization gas from ions with low charge states, for example from the Unresolved Transition Array (UTA) of M-shell iron ( $<$ Fe XVII) at $\sim 16 \AA$ (Sako et al. 2001; Behar et al. 2001) or from inner (K-shell) transitions of $\mathrm{L}$-shell ions from lighter elements, such as $\mathrm{O}$ through to $\mathrm{Si}$ (Behar \& Netzer 2002; Gu et al. 2005). On the other hand, the absorbing gas can also be very highly ionized, in some cases arising from $\mathrm{H}$-like and He-like iron, where such highly ionized gas may originate from an accretion disk wind (e.g., Reeves et al. 2004; Risaliti et al. 2005; Braito et al. 2007; Turner et al. 2008; Tombesi et al. 2010; Gofford et al. 2013). The associated absorption lines are often blueshifted, thus implying outflowing winds, with velocities ranging from a few hundred to a few thousand $\mathrm{km} \mathrm{s}^{-1}$, or even exceeding $10,000 \mathrm{~km} \mathrm{~s}^{-1}$ in the case of the so-called ultra-fast outflows (Chartas et al. 2002; Pounds et al. 2003; Reeves et al. 2003; Gofford et al. 2013; Tombesi et al. 2013, 2015; Longinotti et al. 2015; Nardini et al. 2015).

With the exception of the ultra-fast outflows, which are often highly ionized and may originate from an accretion disk wind on subparsec scales, most soft X-ray warm absorbers are thought to be typically located at fairly large distances from the central black hole. This is a result of their low ionization parameters and outflow velocities $\left(<1000 \mathrm{~km} \mathrm{~s}^{-1}\right)$, their (relative) lack of variability, and in some cases of their inferred low densities (e.g., NGC 3783: Behar et al. 2003; Krongold et al. 2005; Mrk 279: Scott et al. 2004; Ebrero et al. 2010; 
NGC 4051: Steenbrugge et al. 2009; Mrk 290: Zhang et al. 2011; and Mkn 509: Kaastra et al. 2012). These soft X-ray warm absorbers can be associated with, for example, a wind originating from the putative parsec-scale torus (Blustin et al. 2005) or the latter stages of an accretion disk wind that has propagated out to larger radii (Proga \& Kallman 2004; Tombesi et al. 2013), or from gas associated with the AGN narrow-line regions (NLRs) (Crenshaw et al. 2000; Crenshaw \& Kraemer 2000). Although the exact kinematic contribution of the warm absorbers to the total AGN luminosity can be difficult to assess (Crenshaw \& Kraemer 2012), they may still play a key role in shaping the wide-scale properties of AGN host galaxies (Hopkins \& Elvis 2010).

\subsection{The Target: Markarian 1040}

The source Mrk 1040 (also known as NGC 931) is a bright nearby $(z=0.016652$, Huchra et al. 1999) Type 1 Seyfert galaxy. The black hole mass probably is $\log \left(M_{\mathrm{BH}} / M_{\odot}\right)=7.64 \pm 0.40$ (Zhou et al. 2010; De Marco et al. 2013), as estimated from its stellar velocity dispersion (Nelson \& Whittle 1995; Tremaine et al. 2002). In X-rays, Mrk 1040 was observed by ASCA in 1994 August (Reynolds et al. 1995), and the spectral features were interpreted as arising from a warm absorber plus a strong broad fluorescent $\mathrm{Fe} \mathrm{K} \alpha$ emission line (FWHM $\sim 16,000-$ $\left.70,000 \mathrm{~km} \mathrm{~s}^{-1} ; \mathrm{EW}=500 \pm 250 \mathrm{eV}\right)$. It is also bright in the hard X-ray bandpass, having been detected in the 70-month Swift BAT catalog, with a $14-195 \mathrm{keV}$ band flux of $6.1 \times 10^{-11}$ erg cm ${ }^{-2} \mathrm{~s}^{-1}$ (Baumgartner et al. 2013). Mrk 1040 was subsequently observed by XMM-Newton in February 2009 when the source flux was high, with a total exposure of $\sim 84 \mathrm{ks}$. The spectrum revealed all the classic Seyfert 1 characteristics: a soft $\mathrm{X}$-ray excess, a deep warm absorber below $2 \mathrm{keV}$, and a strong Fe $\mathrm{K} \alpha$ line profile (Tripathi et al. 2011). The AGN is also strongly variable on timescales of $10^{4} \mathrm{~s}$, and an energy-dependent lag was claimed from the XMM-Newton observation (Tripathi et al. 2011), similar to the soft X-ray (or negative) lags that have been claimed in other AGN (De Marco et al. 2013). Despite its X-ray brightness, however, which is similar to other bright and nearby Seyfert 1 galaxies, the X-ray spectrum of Mrk 1040 is comparatively understudied and no high-resolution X-ray spectrum has yet been published on this AGN.

The host galaxy of Mrk 1040 is an Sbc spiral galaxy and is viewed at high inclination of $b / a=0.21$ (de Zotti \& Gaskell 1985; Amram et al. 1992). The UV continuum is heavily reddened, which Crenshaw \& Kraemer (2001) suggested was due to dust in the plane of the host galaxy. Based on the width of $\mathrm{H} \beta$, FWHM of $\sim 1830 \mathrm{~km} \mathrm{~s}^{-1}$, it is classified as a narrowline Seyfert 1 (Osterbrock \& Shuder 1982). If the narrowness of the Balmer lines is due to a line-of-sight close to the rotation axis of the black hole or accretion disk (Kraemer et al. 2012), then our view of the AGN in Mrk 1040 may be roughly poleon, which suggests that the AGN is highly inclined with respect to the disk of the host galaxy.

This paper presents the analysis of a series of Chandra High Energy Transmission Grating (HETG) (Weisskopf et al. 2000; Canizares et al. 2005) observations of Mrk 1040 from 2013 October to 2014 March, with a total exposure of $200 \mathrm{ks}$. The observations provide the first reported high spectral resolution observations of this Seyfert 1 galaxy over the X-ray band. Subsequent observations with XMM-Newton were also obtained during 2015, with a total exposure of $175 \mathrm{ks}$ in the RGS; these together with the archival observation from 2009 will be presented
Table 1

Summary of Mrk 1040 Chandra Observations

\begin{tabular}{ccccc}
\hline \hline Obsid & ${\text { Start Date } / \text { Time }^{\mathrm{a}}}$ & Inst & Exposure $(\mathrm{ks})$ & Net Rate s $^{-1}$ \\
\hline 15075 & 2014 Feb 25 04:00:17 & HEG & 28.6 & $0.231 \pm 0.004$ \\
& $\ldots$ & MEG & $\ldots$ & $0.432 \pm 0.004$ \\
15076 & 2013 Oct 19 09:24:53 & HEG & 19.7 & $0.237 \pm 0.005$ \\
& $\ldots$ & MEG & $\ldots$ & $0.444 \pm 0.005$ \\
16571 & 2014 Mar 03 12:26:42 & HEG & 89.6 & $0.240 \pm 0.002$ \\
& $\ldots$ & MEG & $\ldots$ & $0.453 \pm 0.002$ \\
16584 & 2014 Feb 26 01:17:35 & HEG & 60.0 & $0.247 \pm 0.002$ \\
& $\ldots$ & MEG & $\cdots$ & $0.459 \pm 0.003$ \\
\hline Total & $\ldots$ & HEG & 197.8 & $0.238 \pm 0.001$ \\
& $\ldots$ & MEG & 197.8 & $0.457 \pm 0.002$ \\
\hline
\end{tabular}

Note.

${ }^{\text {a }}$ Observation start and end times are in UT.

in a subsequent paper (hereafter paper II). One of the primary goals was to study the warm absorber in this AGN in unprecedented detail and resolution. Here the HETG provides the higher energy coverage of the warm absorber, especially in the $1 \mathrm{keV}$ band and above and covering the Ne through $\mathrm{Fe} \mathrm{K}$-shell lines, with the RGS providing high-sensitivity coverage at lower energies down to $0.3 \mathrm{keV}$.

The paper is organized as follows. In Section 2 we describe the observations and reduction of the HETG spectra. Section 3 is devoted to the spectral fitting of the HETG data, including atomic line detections and identifications as well as the measurements of the kinematics and profiles of the absorption lines. The properties of the emission lines, such as in the iron $\mathrm{K}$-shell band and the He-like triplets, are also presented. Section 4 presents photoionization modeling of the X-ray absorption in the HETG, which, as we show, requires multiple absorption components to cover the wide range of ionization states seen in the absorption spectrum. In Section 5 we discuss the origins and infer some physical properties of the X-ray absorbing media observed in Mrk 1040. The properties of the $\mathrm{X}$-ray gas are then compared to the images and kinematics of the nuclear [O III] emitting gas seen from archival Hubble Space Telescope (HST) observations. As we show, the X-ray absorber appears to show no evidence of outflow to high precision, and the ionized gas may be in part associated with a failed wind located within $\sim 100 \mathrm{pc}$ of the nucleus.

\section{Chandra Observations and Data Reduction}

Chandra observed Mrk 1040 four times between 2013 September 24 to 2014 March 3 (see Table 1 for a summary), with three of the four observations occurring within a one-week period from 2014 February 25 and 2014 March 3. Spectra were extracted with the CIAO package v4.7 (Fruscione et al. 2006). Only the first-order dispersed spectra were considered for the medium-energy grating (MEG) and high-energy grating (HEG), and the \pm 1 orders for each grating were subsequently combined for each sequence. No significant spectral variability was observed between the four sequences, with only modest $\sim 5 \%$ variations in count rate (see Table 1). Therefore the spectra were combined from all four sequences to yield a single first-order spectrum for each of the MEG and HEG, yielding net source count rates of $0.457 \pm 0.002 \mathrm{~s}^{-1}$ (from 0.8 to $5.0 \mathrm{keV}$ ) and $0.238 \pm 0.001 \mathrm{~s}^{-1}$ (from 1.2 to $9.0 \mathrm{keV}$ ), respectively, for a total exposure time of $197.8 \mathrm{ks}$. The total 
counts obtained exceeded 90,000 and 47,000 counts for MEG and HEG, respectively, while the background count rate was negligible. The undispersed zeroth-order image shows no evidence for extended X-ray emission in excess of the telescope point-spread function.

The resulting time-averaged source spectra were subsequently binned at three different levels. These correspond to either the half-width at half-maximum (HWHM) of the spectral resolution (i.e., $\Delta \lambda=10 \mathrm{m \AA}$ and $\Delta \lambda=5 \mathrm{~m} \AA$ bins for MEG and HEG, respectively), the FWHM of the resolution, or to twice the FWHM resolution. We note that at $1 \mathrm{keV}$ (or $12.3984 \AA$ ), the HWHM binning corresponds to velocity intervals of $\Delta v=240$ $\mathrm{km} \mathrm{s}^{-1}$ and $\Delta v=120 \mathrm{~km} \mathrm{~s}^{-1}$ per spectral bin for the MEG and HEG, respectively. The former finer HWHM binning was adopted for the subsequent spectral fitting as well as for obtaining velocity profiles of the lines, while the latter more coarsely binned spectra (FWHM or twice FWHM) were used for an initial inspection of the data and for some of the spectral plots. The MEG and HEG spectra were analyzed over the energy ranges of $0.8-5.0 \mathrm{keV}$ and $1.2-9.0 \mathrm{keV}$, respectively. Below $0.8 \mathrm{keV}$, the signal-to-noise ratio of the MEG data was low as a result of the build-up of contamination over time on the ACIS-S CCDs and was excluded from the subsequent fitting. A cross-normalization constant between the MEG and HEG spectra has been included in all the subsequent fits, but in each case it is consistent with 1.0. We note that the overall fluxes, obtained against an absorbed power-law, correspond to $F_{0.5-2 \mathrm{keV}}=1.1 \times 10^{-11} \mathrm{erg} \mathrm{cm}^{-2} \mathrm{~s}^{-1}$ and $F_{2-10 \mathrm{keV}}=3.7 \times 10^{-11} \mathrm{erg} \mathrm{cm}^{-2} \mathrm{~s}^{-1}$, respectively.

The C-statistic (Cash 1979) was employed in the subsequent spectral fits to the HETG, because although an overall large number of counts were obtained in the observations, toward the lower energy (longer wavelength) end of each grating spectrum the total source counts per bin drops below $N<20$ in some of the spectral bins, which is also true at the centroids of the deepest absorption lines in the spectrum. In the case of $\chi^{2}$ minimization, this would cause a somewhat underestimated continuum level at soft X-ray energies. All fit parameters are given in the rest frame of the quasar at $z=0.016652$ and are stated in energy units; the conversion factor is that $1 \mathrm{keV}$ is equivalent to $12.3984 \AA$ in wavelength. In all of the fits, a Galactic absorption of hydrogen column density of $N_{\mathrm{H}}=6.7 \times 10^{20} \mathrm{~cm}^{-2}$ (Kalberla et al. 2005) was adopted, modeled with the Tuebingen-Boulder absorption model (TBABS in XSPEC), see Wilms et al. (2000) and references therein for the cross sections and abundances used in this model. We note that upon including molecular hydrogen, the Galactic column rises to $N_{\mathrm{H}}=8.84 \times 10^{20} \mathrm{~cm}^{-2}$, e.g., see Willingale et al. (2013), although the spectra above $0.8 \mathrm{keV}$ are not sensitive to the exact value adopted here. Values of $H_{0}=70$ $\mathrm{km} \mathrm{s}^{-1} \mathrm{Mpc}^{-1}$, and $\Omega_{\Lambda_{0}}=0.73$ are assumed throughout and errors are quoted at $90 \%$ confidence $\left(\Delta \chi^{2}=2.7\right)$ for one parameter of interest.

\section{X-Ray Spectral Fitting}

\subsection{The Overall Spectral Form}

Figure 1 shows the observed HETG spectrum of Mrk 1040, where the spectrum has been unfolded through the instrumental response against a power-law model of photon index $\Gamma=2$ in order to produce a fluxed spectrum in $\nu F_{\nu}$ flux units. The spectrum has been plotted at the FWHM spectral resolution and over the wavelength (energy) range from 1.7 to $15.2 \AA$ (or 0.82 to $7.3 \mathrm{keV}$ ) and is plotted in wavelength (in the observed
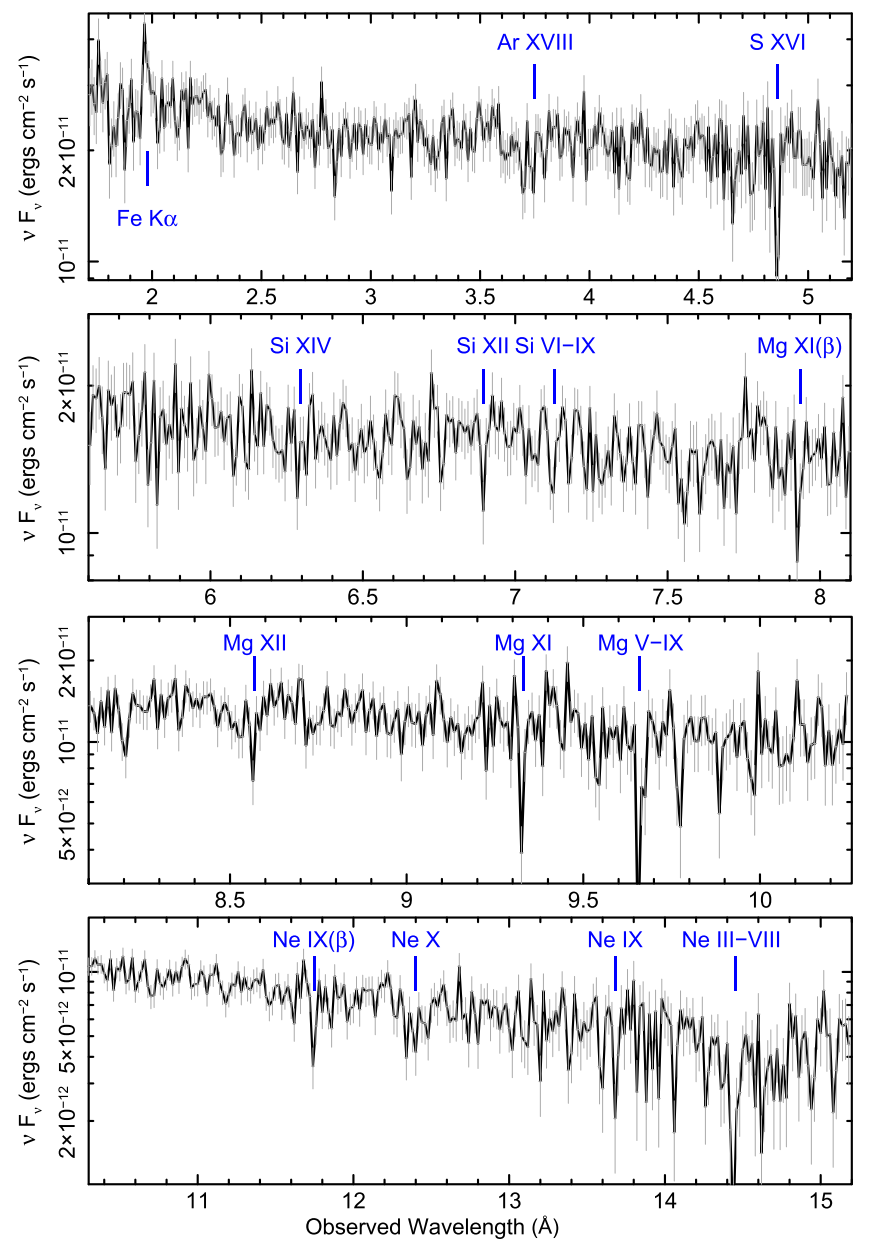

Figure 1. The four panels show the Chandra HETG spectrum of Mrk 1040 in order of increasing wavelength, obtained by combining all four sequences in Table 1. The upper three panels are from the HEG grating and the lower panel is from the MEG. Error bars are shown in gray scale and the spectra are binned at FWHM resolution of $\Delta \lambda=10 \mathrm{m \AA}$ and $\Delta \lambda=20 \mathrm{m \AA}$ for the HEG and MEG, respectively. The spectra have been fluxed against a power-law continuum to create a $\nu F_{\nu}$ spectrum and are plotted in the observed frame. Multiple absorption lines from an ionized absorber are present in the spectrum, as is shown from bottom to top: (i) Ne III-X, (ii) $\mathrm{Mg}$ V-XII, (iii) $\mathrm{Si} \mathrm{VI-XIV,} \mathrm{and}$ (iv) S XVI and Ar XVIII. Thus absorption is seen from ions covering a wide range of ionization, from the low-ionization inner-shell lines of $\mathrm{Ne}, \mathrm{Mg}$, and $\mathrm{Si}$ (see Behar \& Netzer 2002) through to the H-like lines.

frame) to show the overall spectral form. The spectrum shows many clear signatures of a warm absorber, with several K-shell absorption lines present from the most abundant elements, where the strongest lines have been identified in the figure panels. In addition to the highly ionized absorption lines from $\mathrm{He}$ and $\mathrm{H}$-like ions (e.g., from $\mathrm{Ne}, \mathrm{Mg}$, and $\mathrm{Si}$, as well as the $\mathrm{H}$-like lines from $\mathrm{S}$ and $\mathrm{Ar}$ ), evidence for lower ionization absorption is also present. This is in the form of inner K-shell $(1 s \rightarrow 2 p)$ absorption lines from ions corresponding to charge states from Li to F-like (i.e., where the L-shell is partially occupied), see Behar \& Netzer (2002). These can be seen in the spectrum via absorption lines from charge states varying from Ne III-VIII (14.0-14.8 $\AA$ observed frame), Mg V-IX $(9.5-10.0 \AA)$ and from Si VI-XII (6.8-7.2 $\AA$ ). The most prominent of these lines appear from $\mathrm{NeV}$ (C-like $\mathrm{Ne}$ ) and Mg VIII (B-like Mg). This suggests that the warm absorbing gas covers a wide range of ionization, as has been observed in other Seyfert 1 galaxies, e.g., NGC 3783 (Kaspi et al. 2000, 2001; 
Krongold et al. 2003), NGC 5548 (Kaastra et al. 2002; Andrade-Velázquez et al. 2010), Mrk 509 (Yaqoob et al. 2003; Smith et al. 2007), NGC 7469 (Blustin et al. 2007), IC 4329A (Steenbrugge et al. 2005b), NGC 3516 (Holczer \& Behar 2012), MCG-6-30-15 (Lee et al. 2001; Turner et al. 2004), and Mrk 279 (Costantini et al. 2007).

We note that we did not detect absorption that was due to either the low-ionization ions of iron, i.e., in the form of the iron M-shell unresolved transition array (Fe I-XVII UTA, Behar et al. 2001; Sako et al. 2001), or due to oxygen (Gu et al. 2005), as these fall below $0.8 \mathrm{keV}$ where the effective area of the HETG drops rapidly. However, the study of the absorption from these lower energy features will be presented in paper II, which covers the soft X-ray RGS spectrum.

\subsection{Absorption Lines in the HETG Spectrum}

In order to measure the absorption line properties, an initial parameterization of the continuum was adopted. The continuum emission was modified by a power law absorbed by the Galactic column and a neutral partial covering absorber was also included (using the XSPEC model ZPCFABS), whereby a fraction $f_{\text {cov }}$ of the primary X-ray continuum passes through the absorber. This simple model is adopted purely to account for the spectral curvature (decrease in flux) toward lower energies that is due to bound-free absorption, but without accounting for any discrete absorption lines. A full description of the photoionization modeling of the spectrum that accounts for both the absorption lines and the spectral curvature is given in Section 4, and its effect on the overall spectrum is shown in Figure 8. The photon index of the power law was found to be $\Gamma=1.75 \pm 0.02$, while the column of the partial covering absorber was found to be $N_{\mathrm{H}}=4.0_{-0.3}^{+0.9} \times 10^{21} \mathrm{~cm}^{-2}$ with a line-of-sight covering fraction of $f_{\mathrm{cov}}>0.92$, i.e., consistent with a fully covering absorber.

While this simple partial covering model is successful in reproducing the spectral curvature, the overall fit is poor, with a fit statistic of $C / \nu=2592.7 / 2262$ (where $\nu$ is the number of degrees of freedom), which is rejected at $>99.99 \%$ confidence. The residuals to this continuum model in the $\mathrm{Ne}, \mathrm{Mg}$, and $\mathrm{Si}$ bands to the MEG spectrum are shown in Figure 2, while the equivalent residuals in the $\mathrm{Mg}$ and $\mathrm{Si}$ bands to the HEG spectrum are shown in Figure 3. A wealth of absorption line structure is clearly present in the HETG spectrum (independently in both the MEG and HEG gratings) against the continuum model, especially at energies below $2 \mathrm{keV}$ in the $\mathrm{Ne}$, $\mathrm{Mg}$, and Si bands. As expected from the initial broadband spectrum, low-ionization gas appears to be present in the form of a multitude of inner K-shell lines of $\mathrm{Ne}, \mathrm{Mg}$, and $\mathrm{Si}$. We refer to Behar \& Netzer (2002) for a compilation of these innershell lines, and we adopt the known energies (wavelengths) of these lines from this paper in Table 2. These lines have been detected in other grating spectra of Seyfert 1 AGN with high signal-to-noise ratios, such as in NGC 3783 (Blustin et al. 2002; Kaspi et al. 2002), NGC 4151 (Kraemer et al. 2005), Mrk 509 (Kaastra et al. 2011), NGC 3516 (Holczer \& Behar 2012), NGC 4051 (Lobban et al. 2011), NGC 5548 (Steenbrugge et al. 2005a), MR 2251-178 (Reeves et al. 2013), and MCG -6-30-15 (Holczer et al. 2010).

In the Ne band of the Mrk 1040 MEG spectrum, residuals caused by inner-shell absorption lines that are due to Ne III-VII (O-like $\mathrm{Ne}$ to Be-like $\mathrm{Ne}$ ) are present from $0.85-0.90 \mathrm{keV}$ (13.8-14.6 ̊). In addition, absorption from $\mathrm{He}$ and $\mathrm{H}$-like $\mathrm{Ne}$
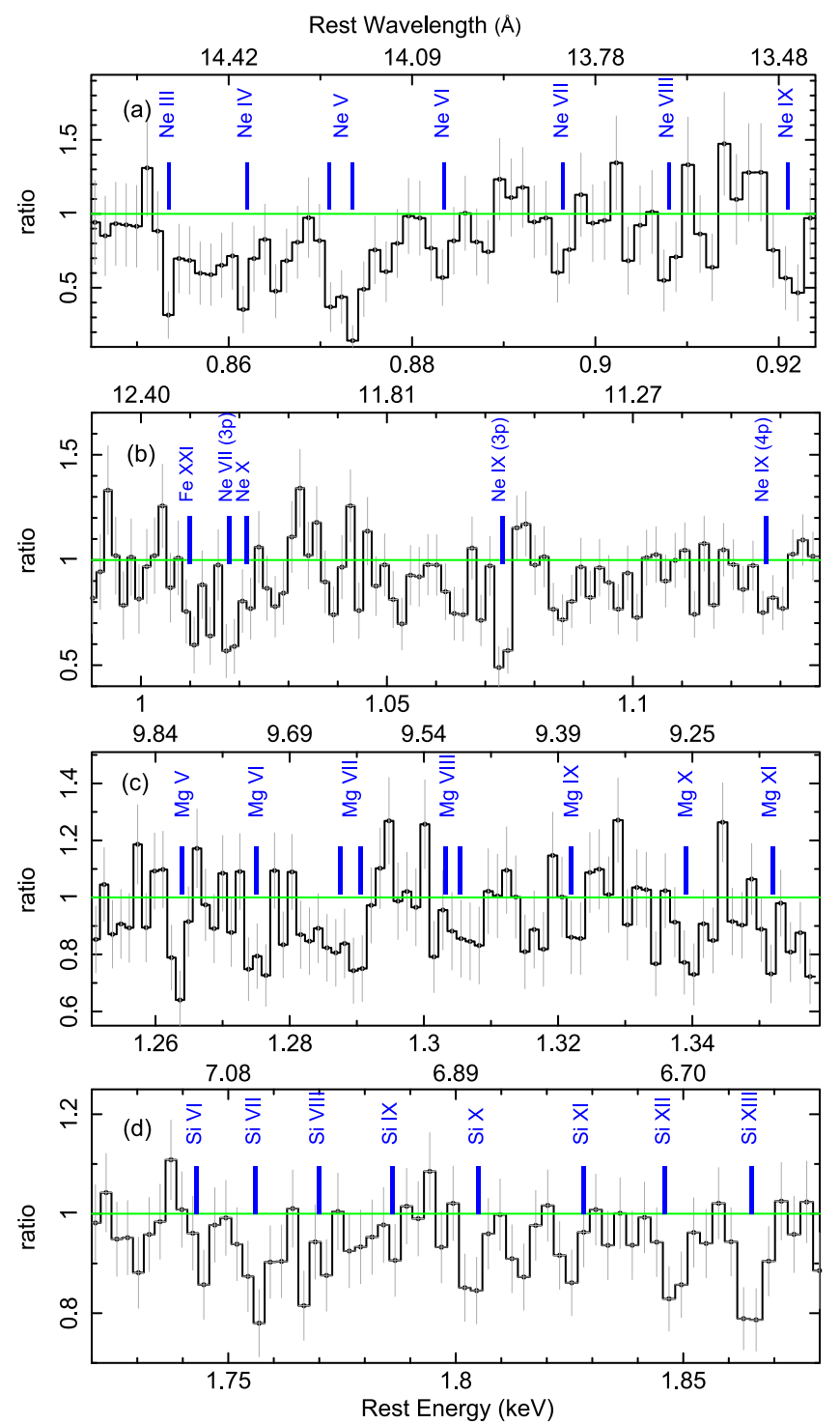

Figure 2. A zoom-in showing the absorption line structure observed in Mrk 1040. The four panels show the data to model residuals to the Chandra MEG spectrum, binned at FWHM resolution, with respect to the baseline continuum consisting of a power law with neutral absorption, as described in Section 3. The panels are plotted in the AGN rest frame (at $z=0.016652$ ), with energy shown on the lower $x$-axis and wavelength on the upper axis. A wealth of absorption lines are seen in the spectrum, from top to bottom: (a) Ne III-IX, (b) Ne IX-X, (c) Mg V-XI, and (d) Si VI-XIII. This shows that like the highly ionized $\mathrm{He}$ and $\mathrm{H}$-like lines, absorption is seen from a range of inner $\mathrm{K}$-shell lines of low to intermediate ionization, varying from $\mathrm{O}$ - or F-like ions to Li-like ions. See Table 2 for details of the lines present in the spectra.

is also present at higher energies, in particular, the He-like line series shows absorption from $1 s \rightarrow 2 p$ to $1 s \rightarrow 4 p$ (see Figure 2, panels a and b). This indicates that the absorber exhibits a wide range of ionization that contributes many different charge states to the absorption spectrum. Likewise, the spectrum in the $\mathrm{Mg}$ and $\mathrm{Si} \mathrm{K}$-shell bands shows similar characteristics. Inner-shell lines from $\mathrm{Mg} \mathrm{V}-\mathrm{IX}$ (O-like to Belike ions) are apparent from $1.26-1.32 \mathrm{keV}$ (9.4-9.8 ̊). Similarly, inner-shell absorption is also detected from Si VIXII (F-like to Li-like) around 1.74-1.85 keV (6.7-7.1 ^). Most of the lines are also independently detected in the HEG (Figure 3) as well as the MEG (Figure 2) spectra. Absorption from the resonance $(1 s \rightarrow 2 p)$ He-like lines of $\mathrm{Mg}$ and $\mathrm{Si}$ is 


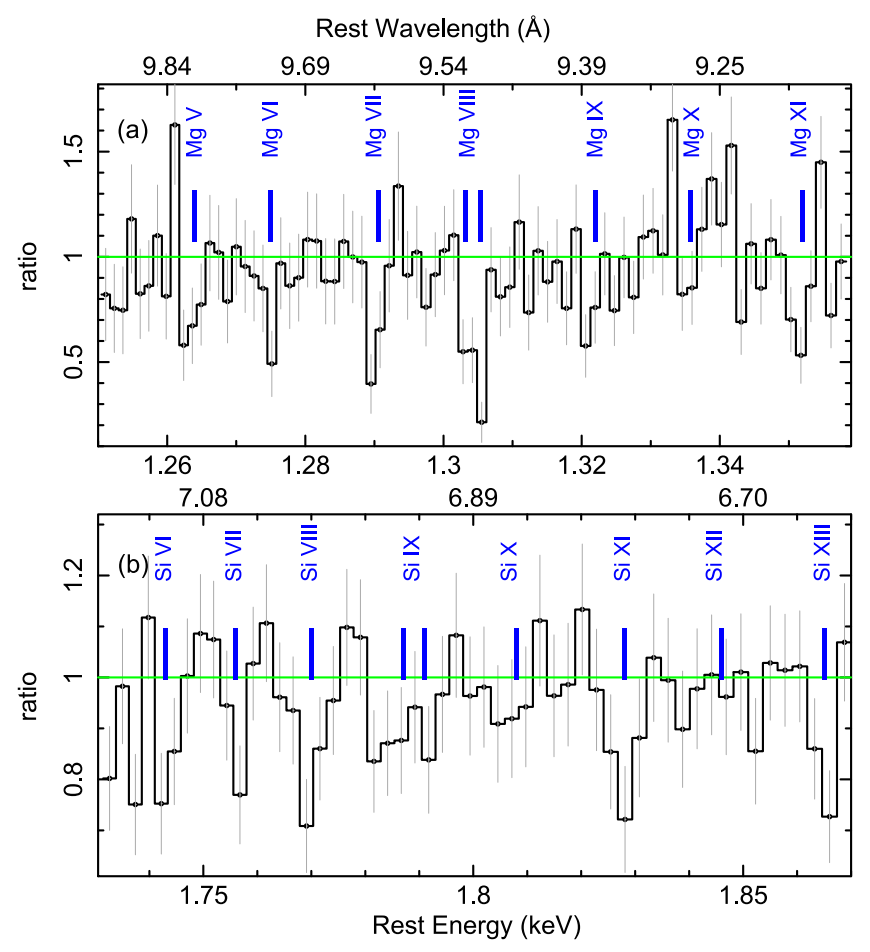

Figure 3. As Figure 2, but showing portions of the Chandra HEG spectrum of Mrk 1040 at FWHM resolution. The upper panel shows the Mg K-shell series of lines, the lower panel the Si K-shell series, each for different ionic charge states. The vertical blue lines mark the expected rest energy (wavelength) of the absorption lines for each charge state from Behar \& Netzer (2002).

also detected (as well as the H-like absorption for $\mathrm{Mg}$ XII), which again confirms that ions from a wide range of charge states are present in the Mrk 1040 absorber.

\subsection{Velocity Profiles}

We then constructed velocity profiles of the principle absorption lines listed in Table 2 . We restricted the analysis to the lines that are detected at least at 95\% confidence (corresponding to $\Delta C=6.0$ for two interesting parameters), noting that most of the lines are detected at least at this level in both gratings (with the exception of the $\mathrm{Ne}$ band, as noted below). A baseline continuum consisting of an absorbed power law was used, for which we employed the neutral partial covering model described in the previous section, as this accounts for the broadband spectral curvature without imparting any discrete absorption lines upon the spectrum. The lines were then fit with Gaussian profiles in the rest energy frame, allowing their centroid energies, widths, and normalizations to vary and accounting for the spectral resolution via the standard instrument responses. To maximize the resolution, HWHM binning was used, and we used the highest resolution HEG data for the $\mathrm{Mg}$ and $\mathrm{Si}$ bands (and above), except for the Ne band, where the MEG was adopted because of the low effective area of the HEG at $1 \mathrm{keV}$ and below. The line profiles were then transposed into velocity space around the known laboratory-frame energy (or wavelength) of each line. When a line profile originated from an unresolved doublet (such as for the $\mathrm{H}$-like lines), we took the weighted mean of the respective laboratory-frame energies to calculate the expected line energy. The doublet corresponding to the $\mathrm{Mg}$ VIII $1 s \rightarrow 2 p$ lines is resolved by the HEG, therefore in that case we fit the two lines separately and obtained the centroid in velocity space separately for each component, i.e., allowing for the different rest-energies of the two lines.

The results of the line profile fitting are summarized in Table 2, which includes 21 line profiles covering 18 separate ionic species from $\mathrm{Ne}$ to Ar. The absorption profiles arise mainly from the $1 s \rightarrow 2 p$ transitions, except for Ne IX and Ne X, where the higher order $1 s \rightarrow 3 p$ lines are detected; in these latter cases, the He- $\beta$ and $\operatorname{Ly} \beta$ profiles provide better constraints on the line widths and velocity centroids because they are free from contamination from other lines. It is also apparent from inspecting the measured energy centroids in Table 2 that most of the lines are positioned close to their expected laboratory-frame energies, while generally the absorption lines are unresolved (or only marginally resolved), with typical velocity widths of $\sigma<300 \mathrm{~km} \mathrm{~s}^{-1}$ (or even $\sigma<100 \mathrm{~km} \mathrm{~s}^{-1}$ in the more tightly constrained cases).

Examples of some of the velocity profiles are plotted in Figure 4 (for the inner-shell and He-like resonance lines) and in Figure 5 (for the H-like lines). In all of the examples, the line centroids are consistent with zero velocity shift within the measurement errors. While it may appear that some of the profiles contain an additional higher velocity component (either red or blueshifted) with respect to the main line centroids, these are due to nearby lines from adjacent ions as labeled in Figures 4 and 5. For example, the strong $\mathrm{NeV}$ absorption profile shows weak absorption components at $\pm 4000 \mathrm{~km} \mathrm{~s}^{-1}$; these are due to neighboring absorption lines from Ne IV and Ne VI at either side of $\mathrm{Ne} \mathrm{V}$ with no velocity shift. This is also the case for the Si VIII and Si XIII profiles, e.g., the latter He-like profile contains a weak contribution from Si XII (Li-like Si) on the red side of the profile. The velocity widths of these profiles are generally low or unresolved, only $\mathrm{NeV}$ is significantly broadened (with $\sigma=490 \pm 200 \mathrm{~km} \mathrm{~s}^{-1}$ ). However, this may be a result of the line being an unresolved doublet (Behar \& Netzer 2002), or from this deep line becoming saturated in its core (as the flux reaches close to zero at the center). In contrast, the He-like profiles of $\mathrm{Ne}$ IX $(\mathrm{He}-\beta)$ and $\mathrm{Mg}$ XI (neither of which are doublets, saturated, or contaminated by other lines) are only marginally resolved, with widths of $\sigma=225_{-100}^{+140} \mathrm{~km} \mathrm{~s}^{-1}$ and $\sigma=150_{-95}^{+110} \mathrm{~km} \mathrm{~s}^{-1}$, respectively. The Mg VIII profile is clearly resolved in the HEG data into a doublet, although the velocity shifts and widths of both components are consistent with zero (e.g., $\sigma<100 \mathrm{~km} \mathrm{~s}^{-1}$ ).

A similar situation emerges for the $\mathrm{H}$-like (Ly $\alpha$ ) profiles, as is shown in Figure 5 for Ne X, Mg XI, S XVI, and Ar XVIII (note that Si XIV falls just below the detection threshold). The Ne X Ly $\alpha$ profile is heavily contaminated by $2 p \rightarrow 3 d$ absorption by Fe XXI, and thus the velocity constraints listed in Table 2 are instead obtained from $\mathrm{Ne} X \mathrm{Ly} \beta$. The highly ionized S XVI and ArXVIII profiles are both unresolved, and their velocity centroids are consistent with zero. Only the Mg XII profile contains some indication of a blueshift. Although the main centroid is consistent with zero velocity, a second velocity component emerges at $-1500 \mathrm{~km} \mathrm{~s}^{-1}$ and appears to be uncontaminated by other lines (note that the separation of the $\mathrm{Mg}$ XII doublet cannot account for this as it is too small to be resolved by the HEG). However, upon inspecting the MEG data for consistency, no such blueshifted component was confirmed, nor is such a component detected in any of the other high-ionization He or H-like lines. Even though this component appears formally significant at the $\sim 99 \%$ level $(\Delta C=10.6)$, as no high-velocity component was measured in any of the other profiles, we do not consider this detection to be plausible. 
Table 2

Gaussian Profiles of Prominent Soft X-Ray Absorption Lines

\begin{tabular}{|c|c|c|c|c|c|c|}
\hline Line ID & $E_{\text {rest }}{ }^{\mathrm{a}}$ & $E_{\mathrm{lab}}^{\mathrm{a}}$ & $v_{\text {out }}^{\mathrm{b}}$ & $\sigma_{\text {width }}{ }^{\mathrm{c}}$ & $\mathrm{EW}(\mathrm{eV})$ & $\Delta C^{\mathrm{d}}$ \\
\hline Ne IV (N-like) & $861.8 \pm 0.6$ & 862.7 & $+310 \pm 210$ & $170_{-120}^{+140}$ & $-1.3 \pm 0.5$ & 8.1 \\
\hline Ne V (C-like) & $873.1 \pm 0.7$ & 873.0 & $-30 \pm 240$ & $490 \pm 200$ & $-3.8_{-0.7}^{+0.9}$ & 30.3 \\
\hline Ne VI (B-like) & $883.3_{-0.8}^{+1.0}$ & 883.4 & $+30_{-340}^{+270}$ & $170^{\mathrm{g}}$ & $-0.9 \pm 0.5$ & 6.7 \\
\hline Ne IX $\mathrm{He}-\alpha$ & $921.5_{-0.8}^{+0.6}$ & 922.0 & $+160_{-195}^{+260}$ & $<520$ & $-1.4_{-0.5}^{+0.8}$ & 13.0 \\
\hline $\mathrm{Ne}$ IX $\mathrm{He}-\beta$ & $1073.4 \pm 0.6$ & 1073.7 & $+80 \pm 170$ & $225_{-100}^{+140}$ & $-1.6_{-0.4}^{+0.5}$ & 21.2 \\
\hline $\operatorname{Ne} x \operatorname{Ly} \alpha^{f}$ & $1018.5_{-0.8}^{+2.7}$ & 1021.5 & $\ldots$ & .. & $-1.7 \pm 0.5$ & 11.5 \\
\hline $\operatorname{Ne} x \operatorname{Ly} \beta$ & $1211.0_{-0.4}^{+0.6}$ & 1210.9 & $-25_{-150}^{+100}$ & $<270$ & $-0.7 \pm 0.3$ & 9.6 \\
\hline Mg V (O-like) & $1264.2 \pm 0.4$ & 1263.1 & $-260 \pm 100$ & $<100$ & $-0.8 \pm 0.4$ & 6.1 \\
\hline Mg VI (N-like) & $1274.9 \pm 0.4$ & 1275.8 & $+210 \pm 100$ & $<100^{\mathrm{g}}$ & $-0.9 \pm 0.4$ & 6.5 \\
\hline Mg VII (C-like) & $1289.4_{-0.3}^{+0.4}$ & 1289.0 & $-90_{-90}^{+70}$ & $<280$ & $-0.9 \pm 0.4$ & 7.1 \\
\hline Mg VIII (B-like) $)^{\mathrm{e}}$ & $1305.6 \pm 0.2$ & 1305.4 & $-50_{-40}^{+45}$ & $<105$ & $-1.5_{-0.2}^{+0.4}$ & 20.7 \\
\hline Mg VIII (B-like) ${ }^{\mathrm{e}}$ & $1303.5_{-0.7}^{+0.5}$ & 1303.2 & $-70_{-115}^{+160}$ & $105^{\mathrm{g}}$ & $-1.0_{-0.4}^{+0.5}$ & 7.7 \\
\hline $\operatorname{Mg}$ XI He- $\alpha$ & $1351.7_{-0.7}^{+0.4}$ & 1352.2 & $+110_{-90}^{+200}$ & $150_{-95}^{+110}$ & $-1.1_{-0.4}^{+0.5}$ & 19.8 \\
\hline $\operatorname{Mg}$ XII Ly $\alpha$ & $1472.0_{-0.8}^{+0.6}$ & 1472.2 & $+40_{-120}^{+160}$ & $150_{-90}^{+140}$ & $-1.1_{-0.5}^{+0.4}$ & 14.6 \\
\hline Si VII (O-like) & $1756.1_{-1.1}^{+0.6}$ & 1755.4 & $-120_{-120}^{+190}$ & $<150$ & $-0.9 \pm 0.5$ & 7.8 \\
\hline Si VIII (N-like) & $1770.3_{-0.7}^{+0.5}$ & 1771.4 & $+185_{-85}^{+120}$ & $<150^{\mathrm{g}}$ & $-1.1_{-0.4}^{+0.5}$ & 12.6 \\
\hline Si XI (Be-like) & $1827.2_{-1.0}^{+1.2}$ & 1829.2 & $+330_{-120}^{+160}$ & $<210$ & $-0.9 \pm 0.4$ & 6.4 \\
\hline Si XII (Li-like) & $1847.1 \pm 0.9$ & 1845.5 & $-260 \pm 150$ & $<210^{\mathrm{g}}$ & $-0.7 \pm 0.4$ & 6.3 \\
\hline Si XIII He- $\alpha$ & $1865.2_{-0.7}^{+0.8}$ & 1865.0 & $-30_{-130}^{+110}$ & $<210^{\mathrm{g}}$ & $-1.2 \pm 0.5$ & 12.4 \\
\hline S XVI Ly $\alpha$ & $2624.1_{-1.9}^{+2.1}$ & 2622.0 & $-240_{-240}^{+220}$ & $<520$ & $-5.4_{-1.7}^{+2.1}$ & 22.5 \\
\hline Ar XVIII Ly $\alpha$ & $3322.6 \pm 1.6$ & 3320.6 & $-180 \pm 150$ & $<950$ & $-3.4_{-1.7}^{+2.3}$ & 8.5 \\
\hline
\end{tabular}

Notes.

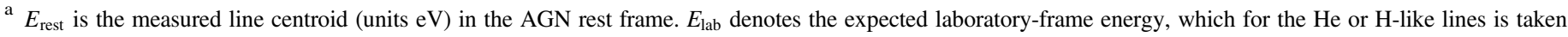

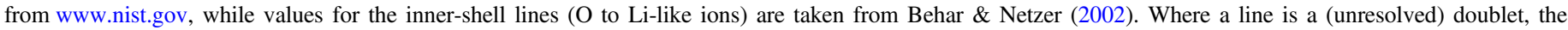
weighted mean of the expected laboratory energies is calculated.

${ }^{\mathrm{b}}$ Velocity shift of absorption line in $\mathrm{km} \mathrm{s}^{-1}$. Negative values denote blueshift, positive values redshift, with the uncertainty range given at $90 \%$ confidence.

${ }^{\mathrm{c}}$ Intrinsic $1 \sigma$ velocity width of the absorption line in $\mathrm{km} \mathrm{s}^{-1}$.

d Improvement in fit statistic after modeling a Gaussian absorption profile.

e Resolved absorption-line doublet modeled by two Gaussian profiles.

${ }^{\mathrm{f}}$ The velocity shift and width of the Ne X Ly $\alpha$ is poorly determined because of the nearby Fe XXI absorption line.

$\mathrm{g}$ Velocity width of line tied to the best-fit value from an adjacent line.

Overall, none of the individual profiles, low ionization as well as high ionization (He and $\mathrm{H}$-like), appear to show a significant outflowing component and most of the lines are unresolved within the instrumental resolution. To determine whether the absorption profiles as a whole are characterized by a small velocity shift, which is within the statistical error of any individual profile, the mean and dispersion of the velocity shifts of the sample of lines was calculated based on the values reported in Table 2. Only one value was considered for each ionic species, taking the best determined values in the three cases where multiple measurements exist for a given ion (e.g., the $\mathrm{Ne} \mathrm{X} 1 s \rightarrow 3 p$ line was favored over the $1 s \rightarrow 2 p$ line). Over 18 ions, the mean velocity shift was found to be $\left\langle v_{\text {out }}\right\rangle=0 \pm 40 \mathrm{~km} \mathrm{~s}^{-1}$, with a dispersion of $\sigma_{v}=180 \mathrm{~km} \mathrm{~s}^{-1}$. Neither was any trend or deviation found between outflow velocity and rest energy. Thus no outflow (or inflow) is required to within $\pm 40 \mathrm{~km} \mathrm{~s}^{-1}$ of the systemic velocity of Mrk 1040 within the absolute wavelength calibration of the HETG gratings. This means that unlike for many other AGN, the Mrk 1040 warm absorber does not appear to originate from an outflow, at least to within very tight limits. In the next section we return to model the absorption with photoionization models generated by XSTAR.

\subsection{Emission Lines}

In contrast to the absorption lines, there are a paucity of soft $\mathrm{X}$-ray emission lines in the HETG spectrum against the bright
$\mathrm{X}$-ray continuum. The emission lines that are detected in the spectrum are listed in Table 3. In the soft X-ray band the most significant emission residuals occur around the $\mathrm{Mg} \mathrm{XI}$ triplet, at the expected positions of the forbidden and intercombination transitions, while an absorption line is present at the resonance line energy, as is shown in Figure 6 for the HEG data. Both emission lines are relatively weak (equivalent width of $1.1 \pm 0.7 \mathrm{eV}$ ) and appear unresolved, with an upper limit to their velocity widths of $\sigma<135 \mathrm{~km} \mathrm{~s}^{-1}$.

The relatively equal contribution of the forbidden and intercombination components appears surprising at first, as this would imply a relatively low ratio of the $R$ value between the two lines $(R=z /(x+y)$, see Porquet \& Dubau 2000) and subsequently a high density of $n_{\mathrm{e}} \sim 10^{13} \mathrm{~cm}^{-3}$. However, given the large errors on the line normalizations, it is not possible to constrain the line ratio, and the derived density is also formally consistent with a lower density photoionized plasma, where $n_{\mathrm{e}}<10^{12} \mathrm{~cm}^{-3}$. Furthermore, a relatively high UV radiation field may also suppress the forbidden line through photoexcitation (Porquet et al. 2010), making the direct measurement of the density from the triplet lines less certain. In addition, the forbidden line may also be absorbed on its blue wing as a result of the adjacent $\mathrm{Mg}$ X resonance absorption line (see Figure 6). We note that no other line emission is detected from the other triplets, e.g., as the signal-to-noise ratio is too low around Ne IX, while the only other soft X-ray line that is 

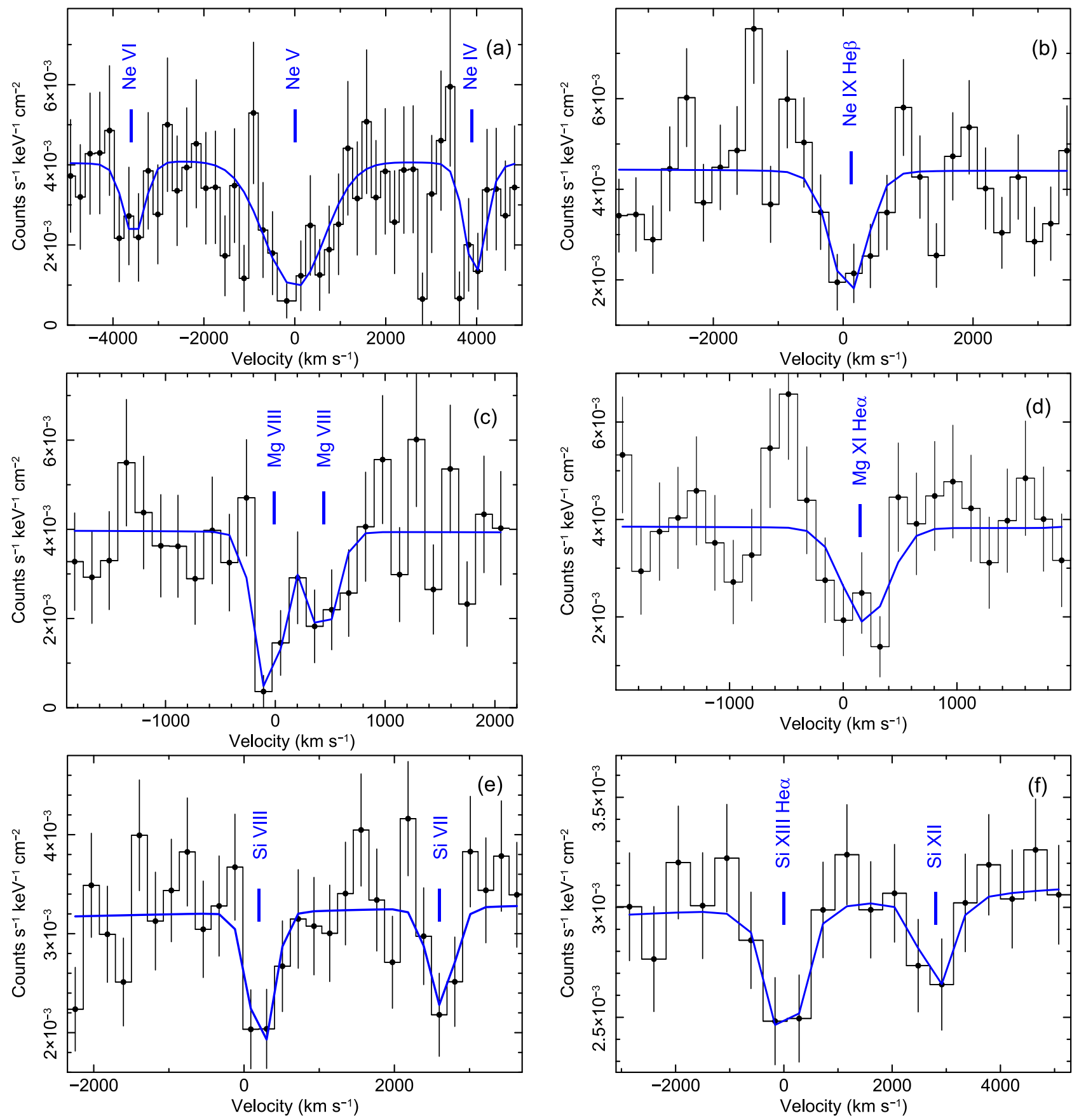

Figure 4. Velocity profiles of selected He-like and inner-shell absorption lines corresponding to (a) $\mathrm{Ne} \mathrm{V,} \mathrm{(b)} \mathrm{Ne}$ IX (He- $\beta$ ), (c) Mg VIII, (d) Mg XI, (e) Si VIII, and (f) $\mathrm{S}$ XIII. The profiles for Ne are extracted from the MEG, while the other lines are extracted from the HEG, at HWHM resolution. Note that negative velocities denote blueshift compared to the expected rest frame energies listed in Table 2. The solid blue lines show the best-fit Gaussian profiles. Other nearby lines from adjacent ions are marked in the plots, while the Mg VIII absorption is from a resolved doublet centered on the stronger of the two lines. Generally, the absorption lines appear centered close to zero velocity (indicating no outflow), with their best-fit line widths and velocity shifts reported in Table 2. See text for a detailed discussion.

marginally detected arises from Si XIV Ly $\alpha$ at $2.0 \mathrm{keV}$, which again is unresolved.

\subsubsection{The Iron K-shell Band}

The strongest signal present in the iron $\mathrm{K}$-shell band arises from the almost ubiquitous narrow $\mathrm{Fe} \mathrm{K} \alpha$ emission line (Nandra et al. 2007); the iron K-band spectrum is plotted in Figure 7. The line is detected at $6403_{-8}^{+12} \mathrm{eV}$ and thus is at the expected energy for near neutral iron. The equivalent width of the narrow $\mathrm{Fe} \mathrm{K} \alpha$ line is $44_{-12}^{+17} \mathrm{eV}$. The line is unresolved, with a formal upper limit to its width of $\sigma<28 \mathrm{eV}$ or $\sigma<1310$ $\mathrm{km} \mathrm{s}^{-1}$ in velocity space. This appears consistent with an origin in distant (e.g., pc scale) gas, which is often found to be case from $\mathrm{Fe} \mathrm{K} \alpha$ line cores seen in other Type I Seyferts as measured by Chandra HETG (Shu et al. 2010). We note that there is some indication of an excess redward of the line core, which is consistent with the presence of a Compton shoulder to the line (George \& Fabian 1991) with an energy of $E=6.33_{-0.07}^{+0.03} \mathrm{keV}$; however, the Compton shoulder is not 

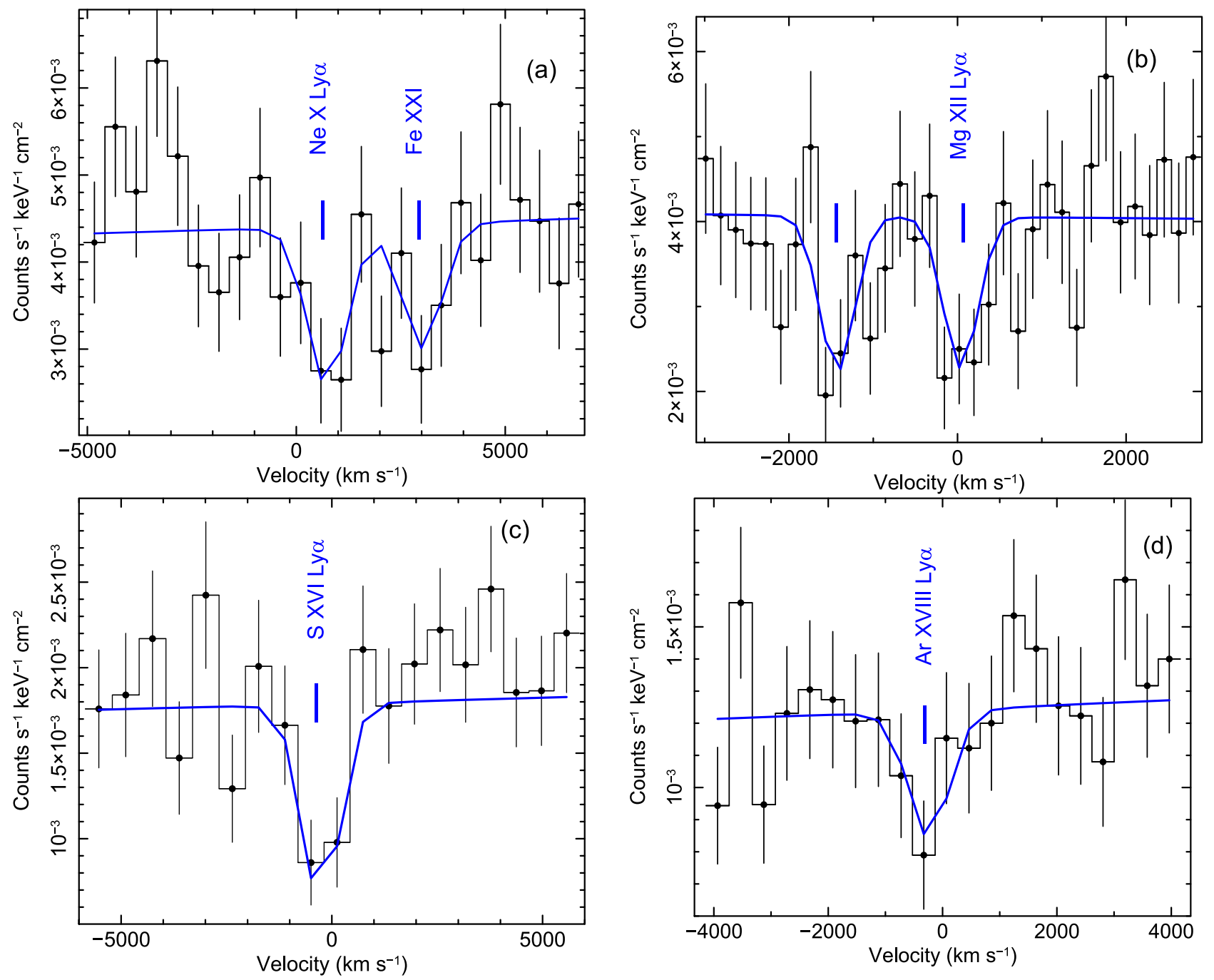

Figure 5. As Figure 4, but showing the velocity profiles of selected H-like (1s-2p) absorption lines corresponding to (a) $\mathrm{Ne}$ X (He- $\alpha$ ), (b) Mg XII, (c) S XVI, and (d) Ar XVIII. The profiles have been extracted from the HEG, with the exception of Ne X (MEG). As for the other profiles, the H-like line profiles generally appear to be unresolved, with outflow velocities consistent with zero (see Table 2). The Mg XII profile does appear to show evidence for a second blueshifted component at $-1400 \mathrm{~km} \mathrm{~s}^{-1}$, but this does not appear to be present in the other H-like profiles, and this component is not confirmed in the MEG data. Note that the Ne X He- $\alpha$ profile contains a contribution from Fe XXI absorption redward of the absorption line profile, and the velocity profile is better determined in the Ly $\beta$ line profile, which is uncontaminated.

Table 3

X-Ray Emission Lines in the Chandra HETG Spectrum of Mrk 1040

\begin{tabular}{llrccr}
\hline \hline Line & $E_{\text {quasar }}{ }^{\mathrm{a}}$ & Flux $^{\mathrm{b}}$ & $\mathrm{EW}^{\mathrm{c}}$ & $\sigma_{\mathrm{v}}{ }^{\mathrm{d}}$ & $\Delta C^{\mathrm{e}}$ \\
\hline $\operatorname{Mg}$ XI $(f)$ & $1333.3 \pm 0.5$ & $0.70_{-0.40}^{+0.45}$ & $1.1 \pm 0.7$ & $<135$ & 9.7 \\
$\operatorname{Mg}$ XI $(i)$ & $1341.2 \pm 0.5$ & $0.70_{-0.39}^{+0.48}$ & $1.1 \pm 0.7$ & $<135^{\mathrm{f}}$ & 9.7 \\
$\operatorname{Si~XIV~Ly~} \alpha$ & $1999_{-8}^{+15}$ & $0.42_{-0.24}^{+0.26}$ & $1.3 \pm 0.8$ & $<390$ & 8.3 \\
Fe K $\alpha$ & $6403_{-8}^{+12}$ & $1.8_{-0.5}^{+0.7}$ & $44_{-12}^{+17}$ & $<1310$ & 35.2 \\
Fe XXV $(f)$ & $6630_{-6}^{+13}$ & $0.70_{-0.49}^{+0.45}$ & $17 \pm 11$ & $<630$ & 6.3 \\
\hline
\end{tabular}

Notes.

${ }^{\mathrm{a}}$ Measured line energy in quasar rest frame, units $\mathrm{eV}$.

${ }^{\mathrm{b}}$ Photon flux, units $\times 10^{-5}$ photons $\mathrm{cm}^{-2} \mathrm{~s}^{-1}$.

${ }^{\mathrm{c}}$ Equivalent width in quasar rest frame, units $\mathrm{eV}$.

d $1 \sigma$ velocity width, units $\mathrm{km} \mathrm{s}^{-1}$.

e Improvement in $\mathrm{C}$-statistic upon adding line to model.

${ }^{\mathrm{f}}$ Indicates parameter is tied.

formally detected ( $\Delta C=4.3)$, and neither is it well constrained. In addition to the possibility of a weak Compton shoulder, there is no evidence for any additional excess emission in the red wing of the line, which may be expected to arise from a broad line from the inner accretion disk. The presence (or lack thereof) of any relativistic line component in Mrk 1040 will be discussed in a forthcoming paper.

In addition to the neutral $\mathrm{K} \alpha$ emission, there is some evidence (at $\Delta C=6.3$ ) for the presence of ionized emission exactly coincident with the expected position of the forbidden line from He-like Fe $(\mathrm{Fe} X X V)$. Although weak $(\mathrm{EW}=17 \pm 11 \mathrm{eV})$, the line centroid is accurately measured to $E=6630_{-6}^{+15} \mathrm{eV}$, consistent with the known energy of the forbidden component, and the line width appears narrow with $\sigma<14 \mathrm{eV}$ (or equivalently $\sigma<630 \mathrm{~km} \mathrm{~s}^{-1}$ ). Its origin therefore most likely arises from distant photoionized material, such as the soft X-ray line emitting and absorbing gas. We note that the residuals at the expected position of the resonance absorption lines from Fe XXV and Fe XXVI (6.70 and $6.97 \mathrm{keV})$ are not formally significant in their own right $(\Delta C=4.4$ and $\Delta C=3.7$, respectively), but in the next section we place a formal limit on any high-ionization absorption component arising from Mrk 1040. 


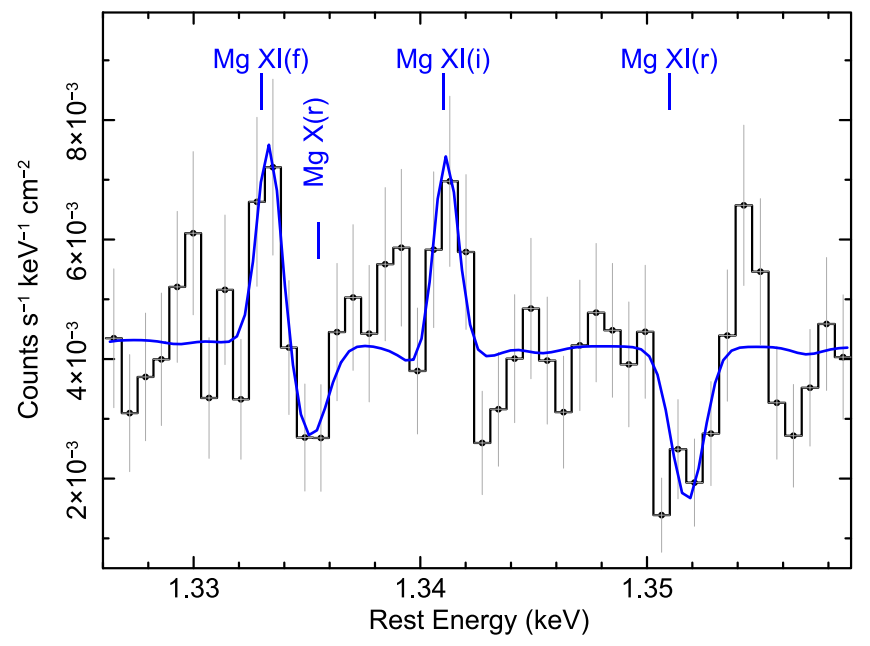

Figure 6. Zoom-in around the Mg XI He-like triplet for the HEG spectrum, binned to HWHM resolution. Count-rate data are shown in black, the model is shown in blue. Excess emission is present near the expected position of the forbidden and intercombination lines, as is marked in the plot, while absorption is clearly observed at the position of the resonance transition. The emission lines are unresolved with a velocity width of $\sigma<135 \mathrm{~km} \mathrm{~s}^{-1}$. Note that the weak absorption immediately blueward of the forbidden emission line is due to the Li-like resonance $(1 s \rightarrow 2 p)$ line of $\mathrm{Mg} \mathrm{X}$.

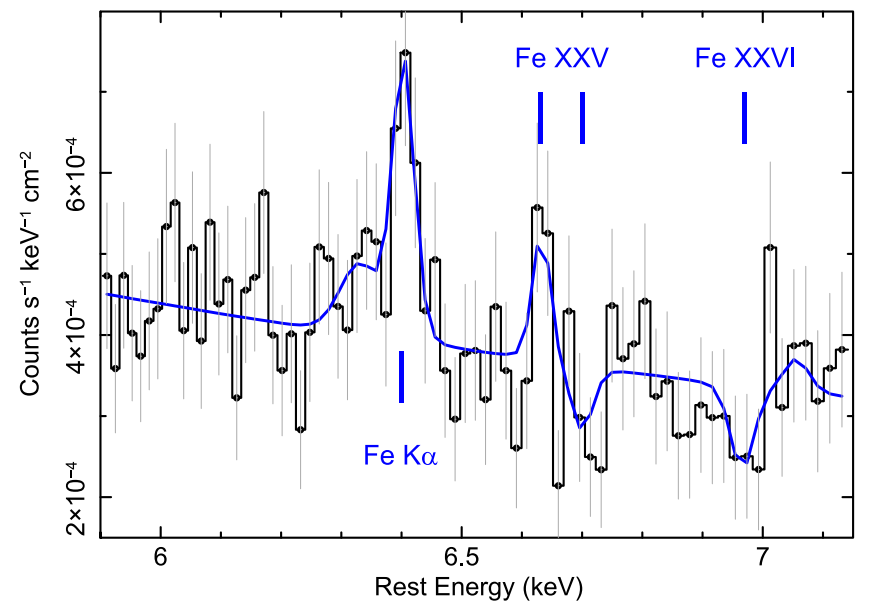

Figure 7. Iron K-band ratio residuals to the HEG spectrum of Mrk 1040 (at HWHM resolution), compared to the best-fit continuum and warm absorber model. The narrow iron $\mathrm{K} \alpha$ line at $6.4 \mathrm{keV}$, with an equivalent width of $44 \mathrm{eV}$, is clearly apparent and is unresolved with a upper limit to its line width of $\sigma<28 \mathrm{eV}$ ( or $<1300 \mathrm{~km} \mathrm{~s}^{-1}$ ). A weak Compton shoulder (in the red wing of the $\mathrm{K} \alpha$ line) may also be present, and the profile is consistent with reflection off distant, near neutral matter. Emission from the forbidden line of $\mathrm{Fe} \mathrm{XXV}$ is present and is unresolved with a line width of $\sigma<14 \mathrm{eV}$ (or $<630 \mathrm{~km} \mathrm{~s}^{-1}$ ) and also likely originates from distant matter. The line contribution of the warm absorber to the iron $\mathrm{K}$ profile is relatively modest, with some weak absorption predicted from the resonance lines of $\mathrm{He}$ and $\mathrm{H}$-like $\mathrm{Fe}$. Note that after accounting for the continuum curvature that is due to the warm absorber, no residuals from a broad emission line are apparent in the Fe $\mathrm{K}$ band.

Finally, in order to determine whether the observed narrow iron $\mathrm{K} \alpha$ emission is consistent with an origin in scattering off distant Compton-thick matter, we instead modeled the spectrum with either a neutral or an ionized Compton-reflection component in addition to the underlying power-law continuum. An excellent fit $(C / \nu=565.2 / 512$ over the $2-8 \mathrm{keV}$ band) was found from modeling the line with a neutral reflection component, adopting the PEXMON model (Nandra et al. 2007) within XSPEC, which returned a reflection fraction of $R=0.30_{-0.12}^{+0.06}$ for an assumed inclination angle of $\theta=45$ degrees and solar abundances. We note that the photon index of the underlying continuum was $\Gamma=1.79_{-0.04}^{+0.07}$. A statistically equivalent fit was also obtained with an ionized reflection model, using the (angle-averaged) tabulated XILLVER model spectra of García \& Kallman (2010) and García et al. (2013), where the upper limit on the ionization parameter was found to be $\log \xi<0.1$, with a consistent photon index of $\Gamma=1.88_{-0.08}^{+0.05}$. The spectrum fit with this model is shown in Figure 7. Thus the $\mathrm{Fe} \mathrm{K} \alpha$ emission appears consistent with an origin from distant Compton-thick matter, and no additional velocity broadening is required to account for this band.

\section{Photoionization Modeling of the X-Ray Absorption Spectrum}

\subsection{Photoionization Models}

Given the substantial presence of partially ionized gas in the X-ray spectrum of Mrk 1040, we attempted to model the absorption spectrum with photoionized grids of models using the XSTAR code v2.2 (Kallman et al. 2004). Absorption grids were generated in the form of XSPEC multiplicative tables. The absorption spectra within each grid were computed between $0.1-20 \mathrm{keV}$ with $N=10,000$ spectral bins. The photoionizing X-ray continuum between 1 and 1000 Rydberg was assumed to be a broken power-law, with a photon index of $\Gamma=2.5$ below a break energy of $0.8 \mathrm{keV}$ (at the low-energy end of the HETG bandpass) and with a photon index of $\Gamma=2$ assumed above this break energy. The 1-1000 Rydberg photoionizing luminosity is predicted to be $L_{\text {ion }} \sim 2 \times 10^{44} \mathrm{erg} \mathrm{s}^{-1}$ for Mrk 1040 using this continuum model. Although the power-law break is not required to model the continuum in the HETG data, which is not sensitive to the lowest energies, it is required by the subsequent XMM-Newton spectra (both for the pn and RGS, paper II) to account for the soft X-ray excess in this source. Furthermore, as we discuss in Section 4.3, a softer photoionizing continuum is required to model the numerous lowionization inner-shell absorption lines in the spectrum, which otherwise are overionized by a flatter $\Gamma=2$ continuum.

With this choice of continuum, we then generated an initial broad grid of models that covered a wide range in ionization and column density: from $N_{\mathrm{H}}=1 \times 10^{18} \mathrm{~cm}^{-2}$ to $N_{\mathrm{H}}=3 \times$ $10^{24} \mathrm{~cm}^{-2}$ and $\log \left(\xi / \mathrm{erg} \mathrm{cm} \mathrm{s}{ }^{-1}\right)=0-5^{12}$ in logarithmic steps of $\Delta\left(\log N_{\mathrm{H}}\right)=0.5$ and $\Delta(\log \xi)=0.5$, respectively. This broad grid was used to provide an initial characterization of the warm absorber zones (i.e., to provide a first-order estimate of the range of ionization and column needed to account for the absorption) and to account for any high-ionization absorption components. A more finely tuned grid (covering a narrower range of parameters) was subsequently generated with the specific purpose of modeling the low-ionization absorption in the Mrk 1040 spectrum, especially the inner-shell lines. The column density of this narrow low-ionization grid covered the range from $N_{\mathrm{H}}=0.5-5.0 \times 10^{21} \mathrm{~cm}^{-2}$ in steps of $\Delta N_{\mathrm{H}}=1 \times 10^{20} \mathrm{~cm}^{-2}$, with the ionization range extending from $\log \left(\xi / \mathrm{erg} \mathrm{cm} \mathrm{s}^{-1}\right)=$ $-1-3$ in 20 steps of $\Delta(\log \xi)=0.2$. A fine spectral resolution of $N=10^{5}$ points over an energy range of $0.1-20 \mathrm{keV}$ was also employed. Given the narrow (or unresolved) widths of the absorption lines detected in the Chandra HETG spectrum, a

\footnotetext{
12 The ionization parameter is defined as $\xi=L_{\text {ion }} / n R^{2}$ (Tarter et al. 1969), where $L_{\text {ion }}$ is the 1-1000 Rydberg ionizing luminosity, $n$ is the electron density, and $R$ is the distance of the ionizing source from the absorbing clouds. The units of $\xi$ are erg $\mathrm{cm} \mathrm{s}^{-1}$.
} 
Table 4

Warm Absorber Parameters from the HETG Spectrum

\begin{tabular}{lllll}
\hline \hline Parameter & Zone 1 & Zone 2 & Zone 3 & Zone 4 \\
\hline$N_{\mathrm{H}}{ }^{\mathrm{a}}$ & $4.0_{-0.4}^{+0.4}$ & $2.1_{-0.3}^{+0.4}$ & $1.5_{-0.4}^{+0.5}$ & $10_{-5}^{+6}$ \\
$\log \left(\xi / \mathrm{erg} \mathrm{cm} \mathrm{s}^{-1}\right)^{\mathrm{b}}$ & $-0.04_{-0.18}^{+0.14}$ & $1.07_{-0.15}^{+0.11}$ & $2.11_{-0.11}^{+0.16}$ & $3.7 \pm 0.4$ \\
$v_{\text {out }}{ }^{\mathrm{c}}$ & $-150_{-100}^{+105}$ & $+10_{-90}^{+80}$ & $+130_{-60}^{+70}$ & $<80$ \\
$\Delta C^{\mathrm{d}}$ & 946.7 & 130.3 & 46.0 & 10.3 \\
\hline
\end{tabular}

Notes.

${ }^{\mathrm{a}}$ Hydrogen column density, units $\times 10^{21} \mathrm{~cm}^{-2}$.

b Ionization parameter in $\log$ units.

${ }^{c}$ Outflow velocity in units $\mathrm{km} \mathrm{s}^{-1}$. Positive values indicate inflow.

${ }^{\mathrm{d}}$ Improvement in C-statistic upon the addition of the component to the model.

turbulence velocity ${ }^{13}$ of $b=100 \mathrm{~km} \mathrm{~s}^{-1}$ was assumed for the absorption models; grids with much higher turbulences all gave substantially worse fits in the models considered below. Solar abundances were adopted for all the abundant elements, using the values of Grevesse \& Sauval (1998), except for Ni, which is set to zero (the default option within XSTAR).

\subsection{Warm Absorber Fits to the Mrk 1040 Chandra HETG Spectrum}

In order to model the absorption spectrum, we successively added individual components of absorbing gas, fully covering the line of sight to the source, until the fit statistic was no longer improved at the $99 \%$ confidence level $(\Delta C=9.2$ for two parameters) and no obvious residuals remained after fitting the spectrum. To fit the continuum itself, we adopted the broken power-law form as described above, with the break energy fixed at $0.8 \mathrm{keV}$ and the photon index below the break also fixed at $\Gamma=2.5$ (as the Chandra data are not sensitive to the slope of the soft excess). We note that the above choice of the continuum is consistent with the later XMM-Newton observation, where the low-energy soft excess is required. Above the break energy, the photon index was allowed to vary along with the overall normalization of the continuum. After adding the required absorption zones as described below, the photon index was found to be $\Gamma=1.78 \pm 0.02$, while after correcting for the absorption, the intrinsic luminosity of Mrk 1040 was $L_{2-10}=2.4 \times 10^{43}$ $\mathrm{erg} \mathrm{s}^{-1}$ and $L_{1-1000 \mathrm{Ryd}}=1.8 \times 10^{44} \mathrm{erg} \mathrm{s}^{-1}$ over the $2-10 \mathrm{keV}$ and 1-1000 Rydberg bands, respectively. The emission lines were also added to the model as Gaussians, as described in Section 3.4; these arise from the $\mathrm{Mg}$ XI triplet, from the $\mathrm{Fe} \mathrm{K} \alpha$ and $\mathrm{Fe}$ XXV components of the iron K-band emission, and a weak emission line from Si XIV Ly $\alpha$ (see Table 3).

Three components of absorbing gas are formally required in the Chandra model to account for the soft X-ray absorption, which are listed as zones 1-3 in Table 4. The finely tuned grid of absorption models was used for all three soft X-ray zones. A fourth highly ionized absorption zone was also added to the model (zone 4, Table 4), using the coarse grid covering a broader range of column and ionization. However, compared to the soft X-ray zones $1-3$, this high-ionization zone is only marginally significant $(\Delta C=11.0)$ and mainly contributes by adding the weak absorption lines due to Fe XXV and Fe XXVI (see Section 3.4.1 and Figure 7). Overall, with all the zones of absorption applied to the continuum, the fit to the Chandra spectrum is very good, with a final fit statistic of $C / \nu=2374.0 / 2252$ and a corresponding

${ }^{13}$ The turbulence velocity width is defined as $b=\sqrt{2} \sigma=$ FWHM $/(2 \sqrt{\ln 2})$.
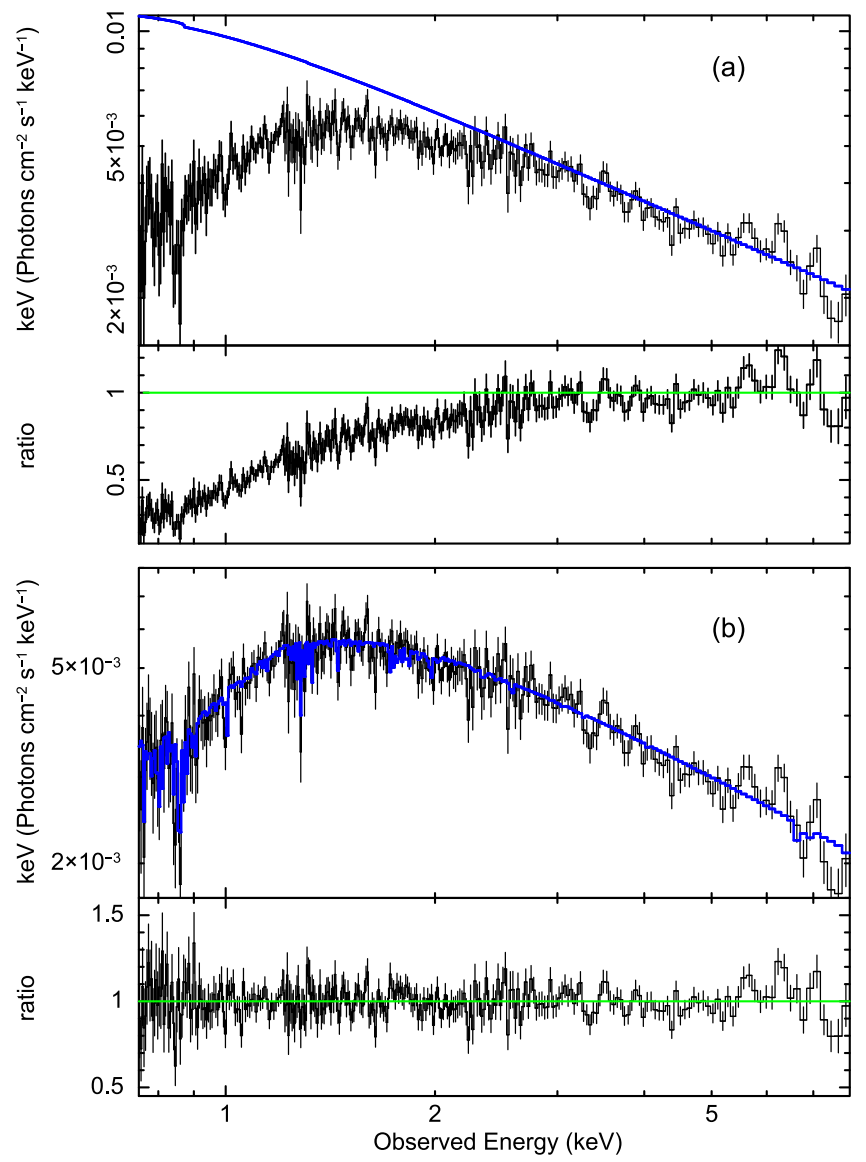

Figure 8. Broadband view of the Chandra HETG spectrum, binned by a factor $\times 2$ the FWHM resolution for clarity. In the upper (a) panel the fluxed spectrum is compared to a simple power-law continuum of $\Gamma=1.8$ fit above $3 \mathrm{keV}$ and attenuated to only the Galactic absorption column. The curvature toward lower energies due to the warm absorber is apparent in the data-to-model ratio residuals. The lower panel (b) shows the spectrum with the best-fit warm absorber model overlaid. In this case, the warm absorber model is able to account for the spectral curvature toward soft X-ray energies. Note that the emission lines have not been included at $\mathrm{Mg}$ or $\mathrm{Fe} \mathrm{K}$ (due to the coarser binning adopted), these are shown in the previous Figures 6 and 7 .

null-hypothesis probability of $P_{\mathrm{f}}=0.16$. In comparison, the fit with the simple neutral partial covering model yielded a substantially worse fit with $C / \nu=2592.7 / 2262$ (as this model only accounts for the continuum curvature, but not the line absorption), while a fit without any absorption zones applied to the continuum (except for the Galactic column) is extremely poor with $C / \nu=3508.3 / 2264$. The effect of the warm absorber upon the overall spectrum is illustrated in Figure 8. Here the top panel shows the spectrum fit with only a power-law continuum (with $\Gamma=1.78$ ) above $3 \mathrm{keV}$, where the pronounced spectral curvature that is due to the warm absorber is apparent toward lower energies. If the warm absorber is applied to the spectral model, then the continuum curvature is clearly accounted for by the absorbing gas (see Figure 8(b)).

Overall, the three soft X-ray warm absorber zones 1-3 that are required to model the Chandra spectrum cover the range in column from $N_{\mathrm{H}}=1.5-4.0 \times 10^{21} \mathrm{~cm}^{-2}$ and ionization from $\log \xi=0-2$ (see Table 4 for detailed parameters), where the lowest ionization zone $1(\log \xi=0)$ has the highest column. As is expected from the velocity profile analysis (Section 3.3), the outflow velocities of the absorption zones are very low and are typically consistent within $\pm 100 \mathrm{~km} \mathrm{~s}^{-1}$ of the systemic 


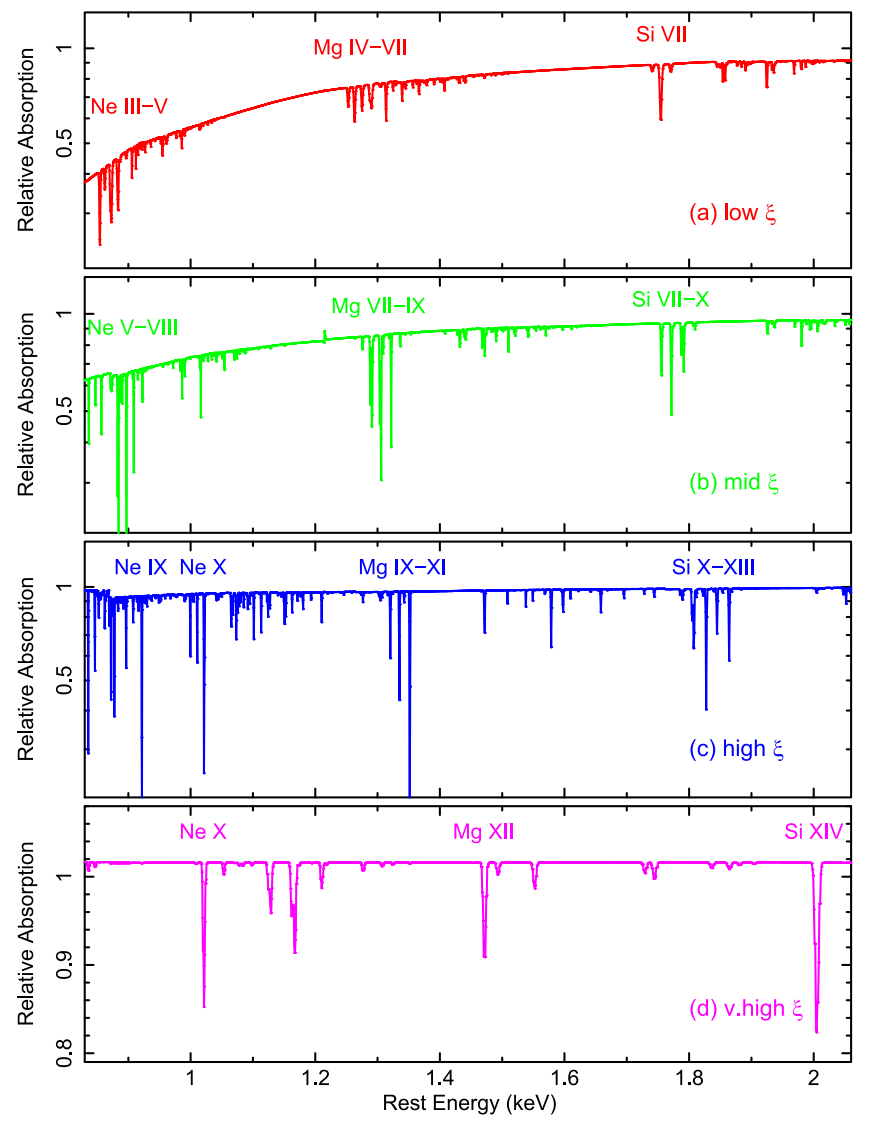

Figure 9. Relative contribution of respective warm absorption zones toward the attenuation of the soft X-ray spectrum. The four absorption components, from lowest to highest ionization, correspond to the warm absorber zones listed in Table 4. The lower ionization zones 1 and 2 (panels a and b) have the highest opacity, with absorption due to inner shell $\mathrm{Ne}, \mathrm{Mg}$, and $\mathrm{Si}$; these zones are responsible for much of the soft X-ray spectral curvature and absorption line structure. The higher ionization zone 3 (panel c) contains absorption that is mainly due to $\mathrm{Li}$ and He-like ions, while the highest ionization zone (panel d) has the lowest opacity and contributes only to the H-like lines.

velocity of Mrk 1040. While the lowest ionization zone 1 hints at some mild outflow (with $v_{\text {out }}=-150_{-100}^{+105} \mathrm{~km} \mathrm{~s}^{-1}$ ), this is not supported by either zones 2 or 3; e.g., zone 2 is consistent with zero $\left(v_{\text {out }}=+10_{-90}^{+80} \mathrm{~km} \mathrm{~s}^{-1}\right)$ and zone 3 suggests very mild inflow (with $v_{\text {out }}=+130_{-60}^{+70} \mathrm{~km} \mathrm{~s}^{-1}$ ). However, any slight difference in velocities between the zones are likely to be within the scatter of the measurements. Even the highly ionized zone 4 (where $\log \xi=3.7$ ) shows no evidence of outflow, with a formal limit on the outflow velocity of $<80 \mathrm{~km} \mathrm{~s}^{-1}$.

Figure 9 shows the relative contributions of each of the warm absorber components against a power-law continuum. The two lower ionization zones 1 and 2 , with $\log \xi=0$ and $\log \xi=1$, respectively (top and middle panels), contribute to the lower ionization ions, reproducing the inner-shell lines of $\mathrm{Ne}, \mathrm{Mg}$, and $\mathrm{Si}$ as seen in the spectrum below $2 \mathrm{keV}$. Most of the bound-free spectral curvature is also imparted upon the spectrum by these two zones, with the lowest ionization zone 1 having the greater opacity toward lower energies. The higher ionization zone 3 (with $\log \xi=2.1$ ) mainly produces the $\mathrm{He}$ and $\mathrm{H}$-like absorption lines from $\mathrm{Ne}, \mathrm{Mg}$, and $\mathrm{Si}$, while the very highly ionized zone 4 is essentially transparent at soft $\mathrm{X}$-rays and only makes a weak contribution to some of the $\mathrm{H}$-like lines. The superposition of these zones is then able to model the absorption line structure seen in the Chandra
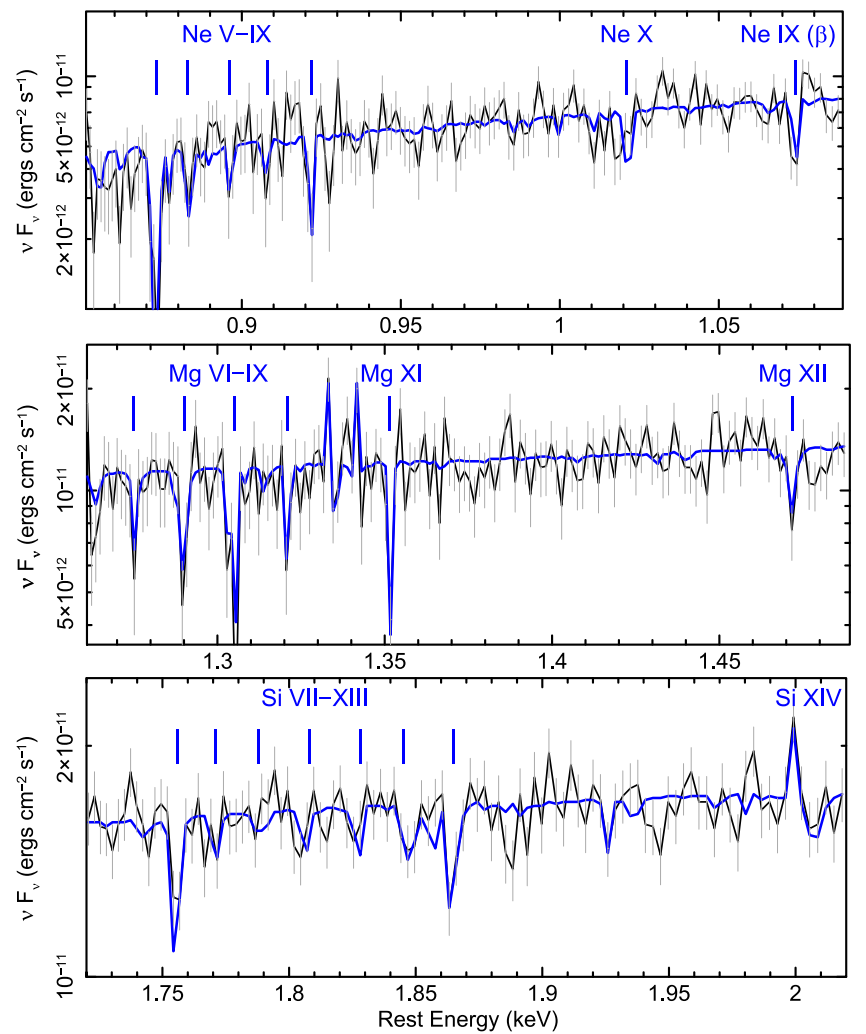

Figure 10. The warm absorber model, shown in blue, superimposed on the HETG spectrum binned at FWHM resolution. The upper panel shows the MEG data and the lower two panels the HEG data. A three-zone warm absorber with ionization in the range $\log \left(\xi / \mathrm{erg} \mathrm{cm} \mathrm{s}^{-1}\right)=0-2$ is able to account for the absorption in the $\mathrm{Ne}$ (upper panel), $\mathrm{Mg}$ (middle panel), and $\mathrm{Si}$ (lower panel) bands, covering the lower ionization inner-shell absorption lines to the highly ionized $\mathrm{He} / \mathrm{H}$-like lines. The warm absorber parameters are tabulated in Table 4, and the net velocities of the zones appear consistent with zero, suggesting that none of the zones appear to be associated with outflowing gas.

spectrum, where Figure 10 shows the best-fit model over the $0.8-2 \mathrm{keV}$ band covering the main absorption lines from $\mathrm{Ne}$, $\mathrm{Mg}$, and Si. The XSTAR model is able to account for the wide range of charge states present, e.g., reproducing the series of absorption lines observed from $\mathrm{Ne} \mathrm{V-X,} \mathrm{Mg} \mathrm{VI-XII,} \mathrm{and} \mathrm{Si} \mathrm{VII-}$ XIII, while simultaneously being able to model for the convex shape of the overall X-ray spectrum.

The broad, and relatively flat distribution of $N_{\mathrm{H}}$ with $\xi$ seen in Table 4 is commensurate with other slow Seyfert outflows; see Behar (2009) where this was parametrized as $N_{\mathrm{H}} \propto \xi^{a}$. Here the value of $a$ corresponds to a density profile of $n \propto r^{-\alpha}$, where $\alpha=(1+2 a) /(1+a)$. Formally, the values in Table 4 (for zones 1-3) yield $a=-0.2 \pm 0.1$ and hence $\alpha=0.8 \pm 0.2$. Such a flat distribution can be explained by an MHD outflow with $n \propto r^{-1}$ (Fukumura et al. 2010), but also with a Radiation-Pressure Compressed (RPC) cloud (Stern et al. 2014). The MHD wind models predict a well-defined line-of-sight outflow velocity structure of $v \propto r^{-1 / 2}$, that for a flat ionization distribution implies $v \propto \xi^{1 / 2}$ that is not quite observed here. The RPC models do not solve for the kinematic structure of the outflow and assume the entire absorber is cruising uniformly at the same velocity (that does not need to be specified for the ionization distribution to be flat). The latter appears to be the case for the absorber of Mrk 1040. Clearly, the broad distribution of $N_{\mathrm{H}}$ with $\xi$ is not consistent with a 
simple radial outflow of $n \propto r^{-2}$, which would naturally produce a narrow distribution of ionization.

\subsection{The Effect of the Ionizing SED on the Absorber}

As discussed above, the shape of the illuminating SED from 1-1000 Ryd may effect the properties of the X-ray absorber via the ionization balance. Here we adopted a broken power-law for the SED, with a steep spectrum below $\sim 1 \mathrm{keV}$, motivated by the need to model the lower ionization lines of $\mathrm{Ne}, \mathrm{Mg}$, and $\mathrm{Si}$ in particular. These inner-shell ions have relatively low ionization potentials for their L-shell electrons, e.g., $266 \mathrm{eV}$ for $\mathrm{Mg}$ VIII and $126 \mathrm{eV}$ for $\mathrm{Ne} \mathrm{V}$; both of the ions produce notable absorption in the spectra, as is shown in Figures 2 and 3. The low ionization potentials of these ions suggest that the form of the EUV and soft X-ray continuum may have an effect on the warm absorber modeling.

In comparison we also tested the effect of a flat $\Gamma=2$ continuum on the absorber properties by replacing the above grids of models with their equivalent versions with the flat SED shape and subsequently minimizing the new spectral fit. In this case, the 1-1000 Rydberg ionizing luminosity is a factor of 3 lower than for the broken power-law case $\left(5.6 \times 10^{43} \mathrm{erg} \mathrm{s}^{-1}\right.$ versus $1.8 \times 10^{44} \mathrm{erg} \mathrm{s}^{-1}$ ). The effect of the flat SED on the subsequent warm absorber modeling is quite apparent, which is illustrated in Figure 11 in the $\mathrm{Ne}$ and Mg K-shell bands. The flat SED model clearly underpredicts the inner-shell absorption from $\mathrm{Mg} \mathrm{V}$-IX and Ne V-VIII, presumably due to the lower luminosity in the EUV to soft X-ray range and because an excess of such photons above the X-ray power law is needed to excite these L-shell electrons. On the other hand, both models are able to account for the higher ionization absorption lines from $\mathrm{He}$ and $\mathrm{H}$-like ions because these are more sensitive to the hard power-law part of the continuum above $1 \mathrm{keV}$ than the soft excess. Overall, the fit with the flat SED model is significantly worse than the softer broken power-law SED model, with $C / \nu=2452.6 / 2252$ versus $C / \nu=2374.0 / 2252$, respectively; here the difference in fit statistic arises from the inability of the former model to account for the lower ionization absorption lines. This demonstrates that the lowionization warm absorber is particularly sensitive to the form of the ionizing continuum in the lowest energy band; this has been found to be the case for the QSOs MR 2251-178 (Reeves et al. 2013) and IRAS 13349+2438 (Laha et al. 2013).

Potentially, the properties of the warm absorber may therefore make it possible to deduce the nature of the soft $\mathrm{X}$-ray continuum and especially the soft excess in AGN. While this is unlikely to be the result of the direct thermal emission from the accretion disk (Gierliński \& Done 2004), some authors have suggested that the origin of the soft excess was atomic and may instead arise from an ionized reflector. An ionized reflection spectrum (Ross \& Fabian 2005), when convolved with the relativistic blurring expected in the innermost disk a few gravitational radii from the black hole, can produce an overall smooth continuum capabale of fitting the featureless soft excess below $1 \mathrm{keV}$ (Crummy et al. 2006; Walton et al. 2013). Alternatively, Comptonization of UV disk photons in a warm optically thick accretion disk atmosphere (Done et al. 2012; Różańska et al. 2015) has also proven to be a plausible form for the EUV to soft X-ray excess in many AGN (Jin et al. 2012; Petrucci et al. 2013; Matt et al. 2014). In Mrk 1040, the warm absorber modeling favors a steep $(\Gamma>2)$ continuum from the EUV to soft X-rays. This might imply that
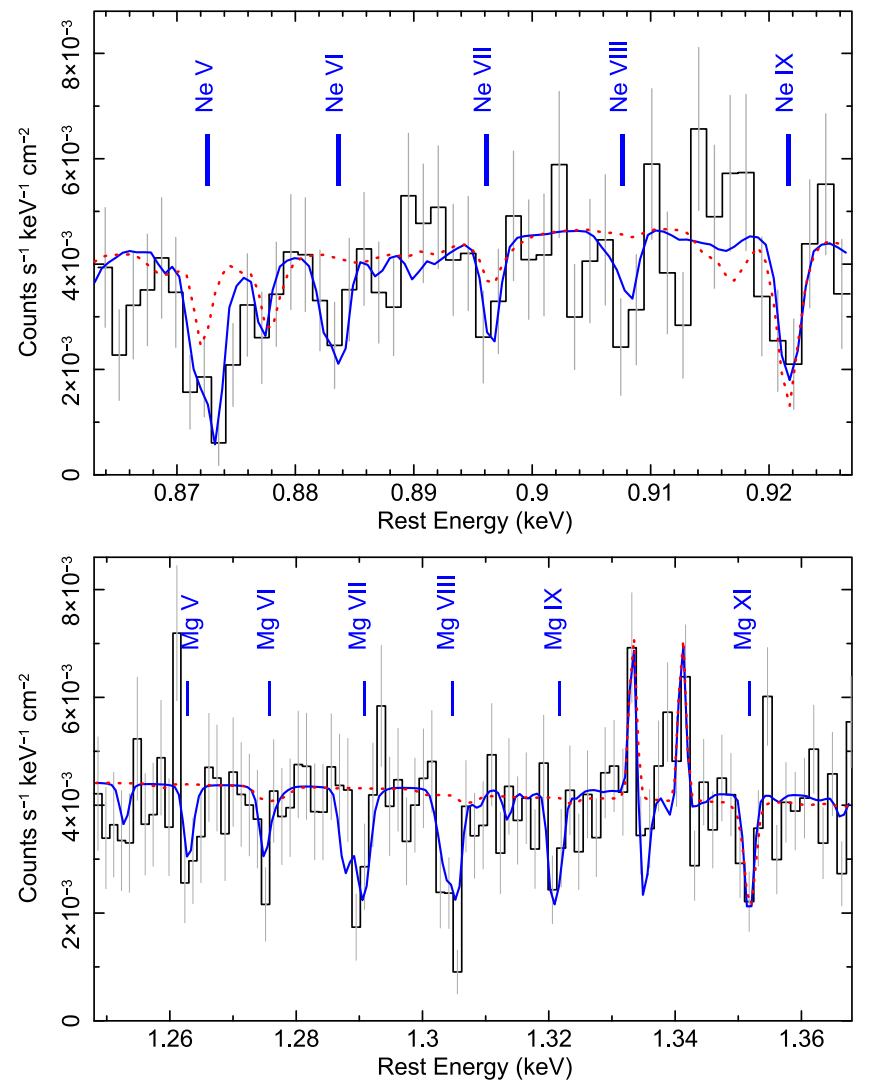

Figure 11. Comparison between warm absorber models in the $\mathrm{Ne}$ (upper panel) and $\mathrm{Mg}$ (lower panel) K-shell bands. The solid blue line shows the best-fit absorber model as described in Section 4.2, which assumes a steep UV to soft $\mathrm{X}$-ray photoionizing continuum of $\Gamma=2.5$ and is able to reproduce all of the absorption features shown. On the other hand, the dotted red line shows an alternative absorber model, where a flat $\Gamma=2$ photoionizing continuum has been assumed from 1 to 1000 Rydberg. This latter model is unable to reproduce the depths of the low-ionization inner-shell absorption lines that are apparent from Ne V-VIII and Mg VI-IX and can only account for high-ionization lines, such as Ne IX or Mg XI. Thus the warm absorber models are sensitive to the assumed slope of the intrinsic soft X-ray continuum. Note the spectra have been binned to the FWHM resolution of the MEG and HEG, respectively.

the soft excess is part of the intrinsic continuum in this AGN and may be produced through disk Comptonization, rather than arising from reprocessed or reflected emission, as is also likely to be the case in MR 2251-178 (Nardini et al. 2014).

\section{Discussion}

\subsection{Main Observational Results}

We have presented the first high-resolution X-ray spectrum of the nearby X-ray bright Seyfert 1 galaxy Markarian 1040. The Chandra HETG observations reveal a spectrum showing a wealth of spectral features from the warm absorber, which covers a wide range in ionization; e.g., from lowly ionized O-like ions to highly ionized $\mathrm{He} / \mathrm{H}$-like like species. The warm absorber parameters for this AGN, with column density in the range from $(1.5<$ $\left.N_{\mathrm{H}}<4.0\right) \times 10^{21} \mathrm{~cm}^{-2}$ and $0<\log \xi<2.1$, are fairly typical for most nearby Seyfert galaxies (Crenshaw et al. 2003; Blustin et al. 2005; McKernan et al. 2007), although at the lower range in ionization. Perhaps the most surprising discovery is the low (or zero) velocity of the gas, consistent within $\pm 100 \mathrm{~km} \mathrm{~s}^{-1}$ of the systemic velocity of Mrk 1040. Thus unlike the majority of Seyfert 1 galaxies, in which the warm absorbing X-ray gas is clearly ascribed to an outflow, the warm absorber in Mrk 1040 
either does not appear to be associated with an outflow, or instead any wind is not able to accelerate out to sufficient distances from the nucleus.

The velocities of most X-ray warm absorber components typically fall in the range from 0 to $-2000 \mathrm{~km} \mathrm{~s}^{-1}$ (Blustin et al. 2005; McKernan et al. 2007; Laha et al. 2014), while the UV velocity components can also cover a similar range of velocity, as seen in X-rays (Crenshaw \& Kraemer 2012). Typical outflow velocities of a few hundred $\mathrm{km} \mathrm{s}^{-1}$ are also seen for the soft X-ray absorber in many well-studied cases, e.g., NGC 3783 (Kaspi et al. 2002) and Mrk 509 (Detmers et al. 2011; Kaastra et al. 2011). Furthermore, Seyfert 1 AGN often show multiple velocity components in the X-ray band, as is also commonly observed in the UV. For example, in an analysis of a deep observation of MCG -6-30-15 observed with Chandra HETG, Holczer et al. (2010) detect a low-velocity absorber with $v_{\text {out }}=-100 \pm 50 \mathrm{~km} \mathrm{~s}^{-1}$, while a higher velocity absorber emerges with $v_{\text {out }}=-1900 \pm 150 \mathrm{~km} \mathrm{~s}^{-1}$ only in the highest ionization component of the warm absorber. In this case, this was directly seen in some of the H-like lines (e.g., Mg XII and Si XIV), where the high-velocity component was revealed in the blue wing of the velocity profiles for these lines. While the lower velocity component of the warm absorber in MCG-6-30-15 appears very similar to what is observed here (with a wealth of inner K-shell and UTA absorption present), no high-velocity component of the highionization lines is seen in Mrk 1040 (e.g., see the velocity profiles in Figures 4 and 5).

The higher ionization zone 3 warm absorber in Mrk 1040 (see Table 4) shows no evidence of outflow $\left(v_{\text {out }}=+130_{-60}^{+70}\right.$ $\mathrm{km} \mathrm{s}^{-1}$ ), while the highest ionization zone 4 (mainly arising from the weak contribution of $\mathrm{He}$ and $\mathrm{H}$-like iron) has an upper limit of $<80 \mathrm{~km} \mathrm{~s}^{-1}$ to the outflow velocity. This is in contrast to the warm absorbers seen in many other Seyferts, where higher velocity zones can emerge in the higher ionization gas. For instance in NGC 3516, fast outflowing zones of up to $-4000 \mathrm{~km} \mathrm{~s}^{-1}$ were present when the AGN continuum brightened (Turner et al. 2008; Holczer \& Behar 2012), which in this case may have coincided with the emergence (or illumination) of an inner disk wind component to the absorber. Similarly, in NGC 4051, both high. (several thousand $\mathrm{km} \mathrm{s}^{-1}$ ) and low-velocity (several hundred $\mathrm{km} \mathrm{s}^{-1}$ ) warm absorbing zones appear to be present (Steenbrugge et al. 2009; Lobban et al. 2011; Pounds \& Vaughan 2011), while in NGC 5548 the onset of a high-velocity (BAL-like) component of the UV and $\mathrm{X}$-ray absorber was also recently revealed in during an absorption episode (Kaastra et al. 2014).

Thus Mrk 1040 appears to be peculiar, in that there is no evidence that the X-ray warm absorber would be outflowing, either through a slow component (with $v_{\text {out }}>100 \mathrm{~km} \mathrm{~s}^{-1}$ ) or a faster higher ionization zone (with $v_{\text {out }}>1000 \mathrm{~km} \mathrm{~s}^{-1}$ ), at least on the basis of the X-ray spectra from the present epoch of observations. Both the low velocity and ionization of the absorber in Mrk 1040 likely place the gas at large distances from the black hole, as we show below. This is far removed from some of the high-velocity and high-ionization components of the warm absorber discussed above, which may instead originate from an accretion disk wind on subparsec scales (e.g., Tombesi et al. 2013) and some of which are powerful enough to drive gas out to kpc scales (Feruglio et al. 2015; Tombesi et al. 2015).

\subsection{The Properties and Location of the Warm Absorber in Mrk 1040}

The best physical constraints on the absorbing gas in Mrk 1040 arise from the zone 3 absorber (see Table 4), as this zone is also associated with the He-like emission from the $\mathrm{Mg}$ triplet, which makes it possible to calculate the covering fraction of the gas as well as its radial location. Photoionized emission spectra in the form of an additive table of models (or an atable within XSPEC) were also generated with XSTAR, with the same properties as the zone 3 absorber and with the same input continuum and ionization $(\log \xi=2.1)$.

From the photoionization modeling, the normalization (or flux), $\kappa$, of an emission component is defined by XSTAR (Kallman et al. 2004) in terms of

$$
\kappa=f \frac{L_{38}}{D_{\mathrm{kpc}}^{2}},
$$

where $L_{38}$ is the ionizing luminosity in units of $10^{38} \mathrm{erg} \mathrm{s}^{-1}$ over the 1-1000 Rydberg band and $D_{\mathrm{kpc}}$ is the distance to the AGN in kpc. ${ }^{14}$ Here $f$ is the covering fraction of the gas with respect to the total solid angle, where $f=\Omega / 4 \pi$ and thus for a spherical shell of gas, $f=1$. For Mrk 1040, where $L_{\text {ion }}=1.8 \times 10^{44} \mathrm{erg} \mathrm{s}^{-1}$ and $D=68.7 \mathrm{Mpc}$ (Theureau et al. 2007), then for a spherical shell the expected XSTAR normalization is $\kappa=3.8 \times 10^{-4}$. Hence for a given column density of gas, this sets the total luminosity of the soft X-ray photoionized emission; also see Reeves et al. (2016) for a similar calculation.

We then applied this XSTAR emission component to the spectrum of Mrk 1040 in order to reproduce the He-like emission from $\mathrm{Mg} \mathrm{XI}$, which is the strongest emission line present in the spectrum and in particular the forbidden line component. When we adopt the above normalization of the emission component, a column density of $N_{\mathrm{H}}=1.0_{-0.5}^{+0.2} \times 10^{21} \mathrm{~cm}^{-2}$ is required, in the case where the gas fully covers the X-ray source with a solid angle of $4 \pi$ steradian. Alternatively, if we fix the column density of the emitter to what was previously measured for the zone 3 absorber (where $N_{\mathrm{H}}=1.5 \times 10^{21} \mathrm{~cm}^{-2}$, see Table 4), then a minimum covering fraction of $f>0.4$ of $4 \pi$ steradian is required to model the emission.

A lower limit on the radial location of the emission can also be obtained from the upper limit to the velocity width of the line emission. When we take $3 \sigma^{2}=G M / R$, and with an upper limit for the velocity width of the $\mathrm{Mg}$ XI forbidden emission of $\sigma_{\mathrm{v}}<135 \mathrm{~km} \mathrm{~s}^{-1}$ (see Table 3), for an estimated black hole mass of Mrk 1040 of $4 \times 10^{7} M_{\odot}$ (Zhou et al. 2010) the location of the emitting gas associated with zone 3 is found to be $>10^{19} \mathrm{~cm}$ or $>3 \mathrm{pc}$.

A typical limit to the radial location of the absorber can be derived from the definition of the ionization parameter and the requirement that the thickness of the absorber does not exceed its distance to the supermassive black hole, i.e., $N_{\mathrm{H}} \simeq n_{\mathrm{H}} \Delta R<n_{\mathrm{H}} R$. Based on the definition of the ionization parameter, this yields

$$
r_{\max } \equiv\left(L_{\text {ion }} / \xi N_{\mathrm{H}}\right)(\Delta R / R) .
$$

Thus for the condition that $\Delta R / R \sim 1$ and for the measured zone 3 absorber parameters of $\log \xi=2.1, \quad N_{\mathrm{H}}=1.5 \times$ $10^{21} \mathrm{~cm}^{-2}$ and an ionizing luminosity of $L_{\text {ion }}=1.8 \times 10^{44}$

\footnotetext{
${ }^{14}$ http://heasarc.gsfc.nasa.gov/docs/software/xstar/docs/html/node96.html
} 
$\operatorname{erg~s}^{-1}$, then $r_{\max } \sim 10^{21} \mathrm{~cm}$ or $<300 \mathrm{pc}$. We note that the location of the gas cannot be much larger than this to satisfy the above geometric condition, while it can be smaller if $\Delta R / R<1$ or if the gas is clumpy.

For comparison, the expected size scale of the NLR can be estimated by the scaling relation of Mor et al. (2009),

$$
R_{\mathrm{NLR}}=295 \times L_{46}^{0.47 \pm 0.13}(\mathrm{pc}),
$$

where here $L_{46}$ is the bolometric luminosity of the AGN in units of $10^{46} \mathrm{erg} \mathrm{s}^{-1}$. If we take the $2-10 \mathrm{keV}$ luminosity to be a typical 3\% of the total bolometric luminosity of Mrk 1040, then $L_{\text {bol }} \sim 7 \times 10^{44} \mathrm{erg} \mathrm{s}^{-1}$ and the estimated distance of the optical NLR would be $R_{\mathrm{NLR}} \sim 100 \mathrm{pc}$, consistent with the above minimum and maximum estimates for the radial distance of the ionized gas (of $3 \mathrm{pc}<R<300 \mathrm{pc}$ ). If the ionized X-ray gas is extended on distances of up to $\sim 300 \mathrm{pc}$, then the density of the gas is low, on the order of $n \sim 1 \mathrm{~cm}^{-3}$ for a column density of $N_{\mathrm{H}}=10^{21} \mathrm{~cm}^{-2}$. This may be expected for largescale gas associated with an extended NLR region. We note that the distance scale of up to $300 \mathrm{pc}$ is consistent with archival HST images (Schmitt et al. 2003), which show extended [O III] emission within $\pm 1^{\prime \prime}$ of the nucleus (see Section 5.3).

We can place upper limits to the mass outflow rate $(\dot{M})$, which in the quasi-spherical case is given by $\dot{M}=f 4 \pi N_{\mathrm{H}} R v_{\text {out }} \mu m_{\mathrm{p}}$, where $f$ is the gas-covering fraction and $\mu \sim 1.2$ is the average particle mass relative to $\mathrm{H}$. From the observations, $N_{\mathrm{H}}=$ $1.5 \times 10^{21} \mathrm{~cm}^{-2}$, the covering fraction (as estimated from the emission) is $f \sim 0.4$, while we adopt a likely upper limit to the outflow velocity of $v_{\text {out }}<100 \mathrm{~km} \mathrm{~s}^{-1}$. Thus for the most likely range in the radial location $(R)$ of the absorber, i.e., from 3 to $300 \mathrm{pc}$ as above, the limits on the mass outflow rate are $\dot{M}<0.02 M_{\odot} \mathrm{yr}^{-1}$ and $\dot{M}<2 M_{\odot} \mathrm{yr}^{-1}$, respectively. Even for the most conservative upper limit of $\dot{M}<2 M_{\odot} \mathrm{yr}^{-1}$, the corresponding upper limit to the kinetic power of the outflow is very low with $L_{\mathrm{K}}<10^{40} \mathrm{erg} \mathrm{s}^{-1}$.

Similar constraints can be placed on the other absorber zones. Given the lower ionizations of zones 1 and 2 (Table 4), Equation (2) would predict $r_{\max }$ values 1-2 orders of magnitude greater than for the more highly ionized zone 3 discussed above (i.e., on kpc scales). However, a moderate clumping of the gas would restrict it to spatial scales consistent with the extended [O III] emitting gas, which is discussed below. Thus for the lowest ionization zone 1 (with $\log \xi=0$ ), if $\Delta R / R \sim 0.02$ and for the measured column of $N_{\mathrm{H}}=4 \times 10^{21} \mathrm{~cm}^{-2}$, then the distance is $r_{\max } \sim 10^{21} \mathrm{~cm} \quad(\sim 300 \mathrm{pc})$ and thus the density would be $n \sim N_{\mathrm{H}} / \Delta R \sim 200 \mathrm{~cm}^{-3}$. This is typical of the estimated densities for line-emitting NLR clouds (Koski 1978). Furthermore, gas with this low ionization $(\log \xi \sim 0$ ) will be more than capable of producing [O III] emission in the optical band. As will be shown from subsequent XMM-Newton/RGS soft X-ray spectroscopy of Mrk 1040 (paper II), absorption is seen in the $\mathrm{O} \mathrm{K}$-shell band covering the full range of charges states; i.e., from $\mathrm{O}$ I-VIII, including the strong detection of K-shell absorption from $\mathrm{O}$ III. The latter absorption is also likely to be associated with the low-ionization zone 1 gas.

\subsection{Imaging and Kinematics of the Nucleus with HST}

Interestingly, from the HST imaging of the [O III] narrowline emitting gas in Mrk 1040 (Schmitt et al. 2003), the NLR appears to be extended on spatial scales of $\sim \pm 1^{\prime \prime}$ from the nucleus. This is consistent with the above maximum estimate of $r_{\max } \sim 300 \mathrm{pc}$ for the warm absorber at the distance of Mrk 1040. Schmitt et al. (2003) report that the [O III] emission appears biconical in structure, with an opening angle of $120^{\circ}$, with the cone axis approximately perpendicular to the host galaxy disk; the HST Wide Field Planetary Camera 2 (WFPC2) image of Mrk 1040 is reproduced in Figure 12 (left panel) from these observations. We note that no direct evidence is found for extended X-ray emission from the Chandra image of Mrk 1040. However, the brightness of the central AGN, the lower spatial resolution of Chandra, and the moderate photon pile-up present in the zeroth-order image precludes a quantitative comparison with the HST image.

Two HST Space Telescope Imaging Spectrometer (STIS) observations of Mrk 1040 in 2009 were performed with the G430M grating, sampling two different position angles through the central part of the galaxy. The details of the observations and of the analysis proceedures are reported by Fischer et al. (2013), who perform imaging and kinematics (primarily in [O III]) of a sample nearby Seyfert galaxies with HST STIS. Here we show the results obtained in Mrk 1040, in order to compare the location and kinematics of the gas emitting in the narrow-line region with the X-ray warm absorber.

These results are summarized in Figure 12. The two STIS observations give two slit positions through different portions of the galaxy, with slit A along the major axis of the galaxy and slit B approximately perpendicular to this. The positions of the two slits are indicated in the left-hand panel of Figure 12, overlaid on the non-dispersed WFPC2 image of Mrk 1040 at [O III] $\lambda 5007$. The center and right panels of Figure 12 show the resulting kinematics of the [O III] line components versus position in arcseconds from the AGN center. These plots indicate that there is evidence of some modest outflow located within \pm 0 ". 2 of the nucleus of Mrk 1040, with outflow velocities of typically $-200 \mathrm{~km} \mathrm{~s}^{-1}$. This blueshifted component of [O III] is visible in both of the slit A and B spectra and occurs well within the inner $100 \mathrm{pc}$ of the central AGN. Slit A, positioned along the major axis, also shows extended blueshifted [O III] emission up to $0 . / 5$ to the southwest of the nucleus and in the direction of the major axis of the galaxy. This emission may be attributed to rotation, if for instance the disk is rotating in a counterclockwise direction and thus the southwest side would appear blueshifted.

\subsection{Implications for the X-Ray Absorber}

The likely distance of the ionized X-ray gas is therefore consistent with the extended emission observed from the optical NLR, as seen in the HST observations. The X-ray kinematics are also broadly consistent with what is seen in $H S T$, where some modest outflow is associated with the nucleus itself of up to $-200 \mathrm{~km} \mathrm{~s}^{-1}$. For the zone 1 absorber, which is most likely associated with the [O III] emission, the outflow velocity is $-150_{-100}^{+105} \mathrm{~km} \mathrm{~s}^{-1}$ (see Table 4). The aperture of Chandra HETG is much larger than for the HST STIS, and thus given the likely upper limit of $<300 \mathrm{pc}$ to the location of the X-ray absorber, we cannot exclude an origin either associated with the weak nuclear outflow located within $<100$ pc of the nucleus as seen in [O III], or with the somewhat more extended gas associated with the rotating galactic disk, with low radial velocities. A tighter constraint on the radial distance of the X-ray absorbing gas would be needed to 

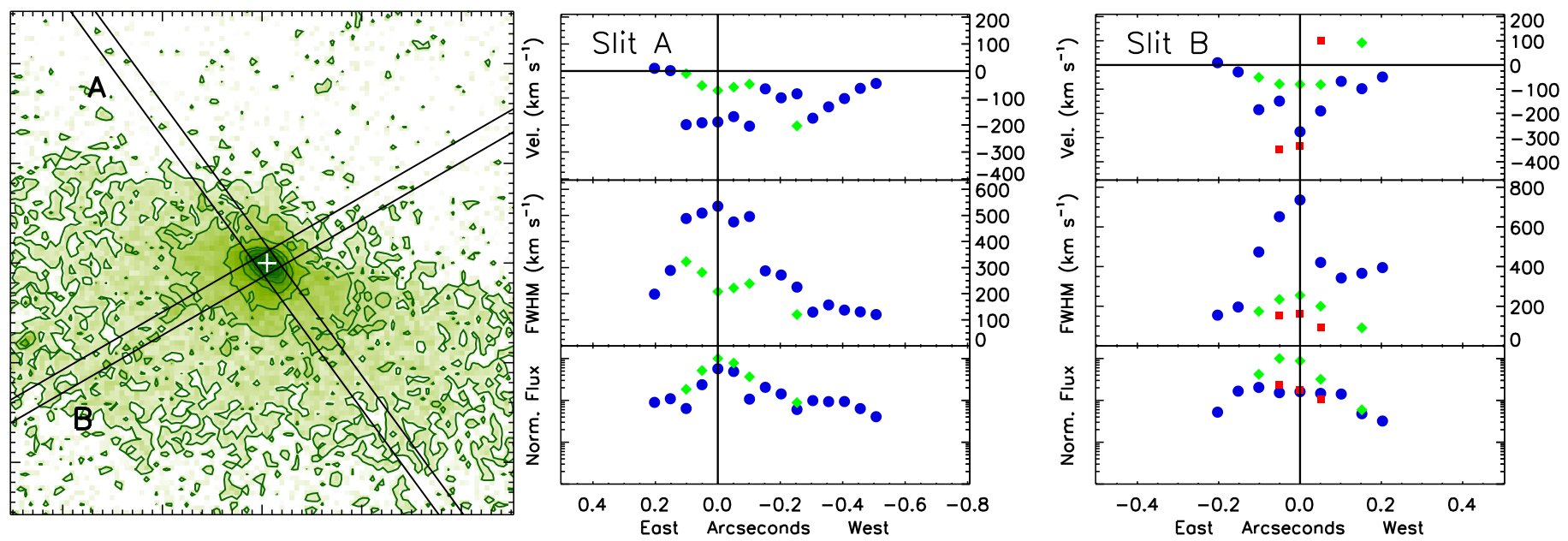

Figure 12. HST imaging observations of the nucleus of Mrk 1040. The left-hand plot shows the WFPC2 image of Mrk 1040 at [O III] $\lambda 5007$ showing the central 2 "! $5 \times 2$ "! 5 region of the galaxy centered on the AGN (white cross). North is up, east is to the left. At the distance of Mrk 1040, the scale is $330 \mathrm{pc}$ arcs ${ }^{-1}$. The position of the two slits used for the HST STIS observations (with the G430M grating) are marked A and B, respectively. Slit A is positioned along the major axis of the galaxy. The middle and right panels show the results of the kinematics obtained from the [O III] line fitting for the two slit positions vs. the position (in arcseconds) from the AGN center. The top panels shows the velocity shift of the [O III] emission vs. position, where negative values denote blueshift. Thus a small component of outflow of $\sim-200 \mathrm{~km} \mathrm{~s}^{-1}$ is found within \pm 0.2 of the nucleus in both of the slit A and B spectra, while a small blueshifted component is seen up to 0 ". $5 \mathrm{SW}$ of the nucleus for slit A only - this latter component may be due to rotation. The middle panels show the FWHM of the [O III] line components we fit, while the lower panel shows the relative [O III] flux vs. position. The different colored points illustrate the kinematics of different components of [O III] selected on FWHM. Thus blue, green, and red points signify the widest, second widest, and third widest components, respectively, as can be seen from their FWHM values in the middle panels.

distinguish between these possibilities; this will be discussed further in paper II, where we present evidence of variations in absorber ionization between the different epochs.

One interesting scenario is that we might be viewing a failed nuclear wind from Mrk 1040. The accretion rate of Mrk 1040, given its luminosity and likely black hole mass, probably does not exceed $10 \%$ of Eddington. With such a weak AGN, it may be difficult to radiatively accelerate gas at distances beyond $\sim 100$ pc (Fischer et al. 2016), although this gas could still be ionized by the AGN. The stringent upper limit obtained earlier on the outflow kinetic power $\left(L_{\mathrm{K}}<10^{40} \mathrm{erg} \mathrm{s}^{-1}\right)$ implies that this weak wind will leave a minimal kinetic footprint on its host galaxy, while the X-ray outflow velocity is also no higher than the velocity dispersion of the stellar bulge in Mrk 1040 (Nelson \& Whittle 1995). The low X-ray velocity may also be partly due to our orientation, if for instance we are viewing Mrk 1040 pole-on compared to a more equatorial wind. The narrowness of the Balmer lines in Mrk 1040 is more likely due to a pole-on view, rather than a high Eddington rate, as is usually inferred in more typical NLS1s.

\section{Conclusions}

This paper has presented a $200 \mathrm{ks}$ Chandra HETG observation of the nearby Seyfert 1 galaxy Mrk 1040, which is the first time that a high-resolution X-ray spectrum of this AGN has been discussed. The spectra show the strong signature of a warm absorber, with the lower ionization gas responsible for the strong series of inner K-shell absorption lines from $\mathrm{Ne}, \mathrm{Mg}$, and $\mathrm{Si}$ originating from ions with low charge states. Neither the low-ionization nor high-ionization components of the absorber appear to be outflowing, to typically within $\pm 100 \mathrm{~km} \mathrm{~s}^{-1}$ of the systemic velocity of Mrk 1040. We also find that the lower ionization (inner K shell) absorption lines are best reproduced with a model that requires a steep $(\Gamma=2.5)$ photon index below $1 \mathrm{keV}$. This argues for an intrinsic origin for the soft X-ray excess from this AGN.
The low ionization and low velocity of the absorber likely place the absorbing gas between $\sim 3$ and $\sim 300 \mathrm{pc}$ from the black hole, and it appears coincident with the optical NLR emitting gas, as seen in [O III] in the HST images of this AGN. This is also broadly similar to the soft X-ray absorbers seen toward other Seyfert 1 galaxies, which may typically range from a parsec-scale toroidal wind (Krolik \& Kriss 2001; Blustin et al. 2005) to the NLR on scales of several tens to hundreds of parsecs (e.g., Behar et al. 2003; Netzer et al. 2003; Kriss et al. 2011; Kaastra et al. 2012). Finally, we suggest that the lack of an X-ray outflow component in Mrk 1040 may be due to a failed nuclear wind in this AGN, with the gas not able to accelerate out to scales beyond $\sim 100 \mathrm{pc}$.

We thank the anonymous referee for their positive and constructive comments. J.N.R. acknowledges Chandra grant number GO3-14123X, as well as NASA grant numbers NNX16AE11G and NNX15AF12G. T.J.T. acknowledges NASA grant number NNH13CH63C. Both J.N.R. and A.L. acknowedge support from STFC, via the consolidated grants ST/M001040/1 and ST/K001000/1. D.P. acknowledges financial support from the European Union Seventh Framework Program (FP7/2007-2013) under grant agreement number 312789. E.B. is supported by the the European Unions Horizon 2020 research and innovation programme under the Marie Sklodowska-Curie grant agreement no. 655324 and by the I-CORE program of the Planning and Budgeting Committee (grant number 1937/12). E.N. also acknowledges funding from the European Unions Horizon 2020 research and innovation programme under the Marie Skodowska-Curie grant agreement No. 664931. T.C.F. was supported by an appointment to the NASA Postdoctoral Program at the NASA Goddard Space Flight Center, administered by Universities Space Research Association under contract with NASA. The scientific results reported in this article are based on observations made by the Chandra X-ray Observatory. This research has made use of 
software provided by the Chandra X-ray Center (CXC) in the application package CIAO.

\section{References}

Amram, P., Marcelin, M., Bonnarel, F., et al. 1992, A\&A, 263, 69 Andrade-Velázquez, M., Krongold, Y., Elvis, M., et al. 2010, ApJ, 711, 888 Baumgartner, W. H., Tueller, J., Markwardt, C. B., et al. 2013, ApJS, 207, 19 Behar, E. 2009, ApJ, 703, 1346

Behar, E., \& Netzer, H. 2002, ApJ, 570, 165

Behar, E., Rasmussen, A. P., Blustin, A. J., et al. 2003, ApJ, 598, 232

Behar, E., Sako, M., \& Kahn, S. M. 2001, ApJ, 563, 497

Blustin, A. J., Branduardi-Raymont, G., Behar, E., et al. 2002, A\&A, 392, 453

Blustin, A. J., Kriss, G. A., Holczer, T., et al. 2007, A\&A, 466, 107

Blustin, A. J., Page, M. J., Fuerst, S. V., Branduardi-Raymont, G., \& Ashton, C. E. 2005, A\&A, 431, 111

Braito, V., Reeves, J. N., Dewangan, G. C., et al. 2007, ApJ, 670, 978

Canizares, C. R., Davis, J. E., Dewey, D., et al. 2005, PASP, 117, 1144

Cash, W. 1979, ApJ, 228, 939

Chartas, G., Brandt, W. N., Gallagher, S. C., \& Garmire, G. P. 2002, ApJ, 579, 169

Costantini, E., Kaastra, J. S., Arav, N., et al. 2007, A\&A, 461, 121

Crenshaw, D. M., \& Kraemer, S. B. 2000, ApJL, 532, L101

Crenshaw, D. M., \& Kraemer, S. B. 2001, ApJL, 562, L29

Crenshaw, D. M., \& Kraemer, S. B. 2012, ApJ, 753, 75

Crenshaw, D. M., Kraemer, S. B., \& George, I. M. 2003, ARA\&A, 41, 117

Crenshaw, D. M., Kraemer, S. B., Hutchings, J. B., et al. 2000, AJ, 120, 1731

Crummy, J., Fabian, A. C., Gallo, L., \& Ross, R. R. 2006, MNRAS, 365, 1067

De Marco, B., Ponti, G., Cappi, M., et al. 2013, MNRAS, 431, 2441

Detmers, R. G., Kaastra, J. S., Steenbrugge, K. C., et al. 2011, A\&A, 534, A38

de Zotti, G., \& Gaskell, C. M. 1985, A\&A, 147, 1

Done, C., Davis, S. W., Jin, C., Blaes, O., \& Ward, M. 2012, MNRAS, 420,1848

Ebrero, J., Costantini, E., Kaastra, J. S., et al. 2010, A\&A, 520, A36

Feruglio, C., Fiore, F., Carniani, S., et al. 2015, A\&A, 583, A99

Fischer, T. C., Crenshaw, D. M., Kraemer, S. B., \& Schmitt, H. R. 2013, ApJS, 209, 1

Fischer, T. C., Machuca, C., Diniz, M. R., et al. 2017, ApJ, 834, 30

Fruscione, A., McDowell, J. C., Allen, G. E., et al. 2006, Proc. SPIE, 6270, $62701 \mathrm{~V}$

Fukumura, K., Kazanas, D., Contopoulos, I., \& Behar, E. 2010, ApJ, 715, 636

García, J., Dauser, T., Reynolds, C. S., et al. 2013, ApJ, 768, 146

García, J., \& Kallman, T. R. 2010, ApJ, 718, 695

George, I. M., \& Fabian, A. C. 1991, MNRAS, 249, 352

George, I. M., Turner, T. J., Netzer, H., et al. 1998, ApJS, 114, 73

Gierliński, M., \& Done, C. 2004, MNRAS, 349, L7

Gofford, J., Reeves, J. N., Tombesi, F., et al. 2013, MNRAS, 430, 60

Grevesse, N., \& Sauval, A. J. 1998, SSRv, 85, 161

Gu, M. F., Schmidt, M., Beiersdorfer, P., et al. 2005, ApJ, 627, 1066

Holczer, T., \& Behar, E. 2012, ApJ, 747, 71

Holczer, T., Behar, E., \& Arav, N. 2010, ApJ, 708, 981

Hopkins, P. F., \& Elvis, M. 2010, MNRAS, 401, 7

Huchra, J. P., Vogeley, M. S., \& Geller, M. J. 1999, ApJS, 121, 287

Jin, C., Ward, M., Done, C., \& Gelbord, J. 2012, MNRAS, 420, 1825

Kaastra, J. S., de Vries, C. P., Steenbrugge, K. C., et al. 2011, A\&A, 534, A37

Kaastra, J. S., Detmers, R. G., Mehdipour, M., et al. 2012, A\&A, 539, A117

Kaastra, J. S., Kriss, G. A., Cappi, M., et al. 2014, Sci, 345, 64

Kaastra, J. S., Mewe, R., Liedahl, D. A., Komossa, S., \& Brinkman, A. C. 2000, A\&A, 354, L83

Kaastra, J. S., Steenbrugge, K. C., Raassen, A. J. J., et al. 2002, A\&A, 386, 427 Kalberla, P. M. W., Burton, W. B., Hartmann, D., et al. 2005, A\&A, 440, 775

Kallman, T. R., Palmeri, P., Bautista, M. A., Mendoza, C., \& Krolik, J. H. 2004, ApJS, 155, 675

Kaspi, S., Brandt, W. N., George, I. M., et al. 2002, ApJ, 574, 643

Kaspi, S., Brandt, W. N., Netzer, H., et al. 2000, ApJL, 535, L17

Kaspi, S., Brandt, W. N., Netzer, H., et al. 2001, ApJ, 554, 216

Koski, A. T. 1978, ApJ, 223, 56

Kraemer, S. B., Crenshaw, D. M., Dunn, J. P., et al. 2012, ApJ, 751, 84

Kraemer, S. B., George, I. M., Crenshaw, D. M., et al. 2005, ApJ, 633, 693

Kriss, G. A., Arav, N., Kaastra, J. S., et al. 2011, A\&A, 534, A41

Krolik, J. H., \& Kriss, G. A. 2001, ApJ, 561, 684
Krongold, Y., Nicastro, F., Brickhouse, N. S., et al. 2003, ApJ, 597, 832

Krongold, Y., Nicastro, F., Brickhouse, N. S., Elvis, M., \& Mathur, S. 2005, ApJ, 622, 842

Laha, S., Dewangan, G. C., Chakravorty, S., \& Kembhavi, A. K. 2013, ApJ, 777,2

Laha, S., Guainazzi, M., Dewangan, G. C., Chakravorty, S., \& Kembhavi, A. K. 2014, MNRAS, 441, 2613

Lee, J. C., Ogle, P. M., Canizares, C. R., et al. 2001, ApJL, 554, L13

Lobban, A. P., Reeves, J. N., Miller, L., et al. 2011, MNRAS, 414, 1965

Longinotti, A. L., Krongold, Y., Guainazzi, M., et al. 2015, ApJL, 813, L39

Matt, G., Marinucci, A., Guainazzi, M., et al. 2014, MNRAS, 439, 3016

McKernan, B., Yaqoob, T., George, I. M., \& Turner, T. J. 2003, ApJ, 593,142

McKernan, B., Yaqoob, T., \& Reynolds, C. S. 2007, MNRAS, 379, 1359

Mor, R., Netzer, H., \& Elitzur, M. 2009, ApJ, 705, 298

Nandra, K., O’Neill, P. M., George, I. M., \& Reeves, J. N. 2007, MNRAS, 382, 194

Nardini, E., Reeves, J. N., Gofford, J., et al. 2015, Sci, 347, 860

Nardini, E., Reeves, J. N., Porquet, D., et al. 2014, MNRAS, 440, 1200

Nelson, C. H., \& Whittle, M. 1995, ApJS, 99, 67

Netzer, H., Kaspi, S., Behar, E., et al. 2003, ApJ, 599, 933

Osterbrock, D. E., \& Shuder, J. M. 1982, ApJS, 49, 149

Petrucci, P.-O., Paltani, S., Malzac, J., et al. 2013, A\&A, 549, A73

Porquet, D., \& Dubau, J. 2000, A\&AS, 143, 495

Porquet, D., Dubau, J., \& Grosso, N. 2010, SSRv, 157, 103

Porquet, D., Reeves, J. N., O’Brien, P., \& Brinkmann, W. 2004, A\&A, 422, 85

Pounds, K. A., Reeves, J. N., King, A. R., et al. 2003, MNRAS, 345, 705

Pounds, K. A., \& Vaughan, S. 2011, MNRAS, 413, 1251

Proga, D., \& Kallman, T. R. 2004, ApJ, 616, 688

Reeves, J. N., Nandra, K., George, I. M., et al. 2004, ApJ, 602, 648

Reeves, J. N., O’Brien, P. T., \& Ward, M. J. 2003, ApJL, 593, L65

Reeves, J. N., Porquet, D., Braito, V., et al. 2013, ApJ, 776, 99

Reeves, J. N., Porquet, D., Braito, V., et al. 2016, ApJ, 828, 98

Reynolds, C. S. 1997, MNRAS, 286, 513

Reynolds, C. S., Fabian, A. C., \& Inoue, H. 1995, MNRAS, 276, 1311

Risaliti, G., Bianchi, S., Matt, G., et al. 2005, ApJL, 630, L129

Różańska, A., Malzac, J., Belmont, R., Czerny, B., \& Petrucci, P.-O. 2015, A\&A, 580, A77

Ross, R. R., \& Fabian, A. C. 2005, MNRAS, 358, 211

Sako, M., Kahn, S. M., Behar, E., et al. 2001, A\&A, 365, L168

Schmitt, H. R., Donley, J. L., Antonucci, R. R. J., Hutchings, J. B., \& Kinney, A. L. 2003, ApJS, 148, 327

Scott, J. E., Kriss, G. A., Lee, J. C., et al. 2004, ApJS, 152, 1

Shu, X. W., Yaqoob, T., \& Wang, J. X. 2010, ApJS, 187, 581

Smith, R. A. N., Page, M. J., \& Branduardi-Raymont, G. 2007, A\&A, 461, 135

Steenbrugge, K. C., Fenovčík, M., Kaastra, J. S., Costantini, E., \& Verbunt, F. 2009, A\&A, 496, 107

Steenbrugge, K. C., Kaastra, J. S., Crenshaw, D. M., et al. 2005a, A\&A, 434, 569

Steenbrugge, K. C., Kaastra, J. S., Sako, M., et al. 2005b, A\&A, 432, 453

Stern, J., Behar, E., Laor, A., Baskin, A., \& Holczer, T. 2014, MNRAS, 445,3011

Tarter, C. B., Tucker, W. H., \& Salpeter, E. E. 1969, ApJ, 156, 943

Theureau, G., Hanski, M. O., Coudreau, N., Hallet, N., \& Martin, J.-M. 2007, A\&A, 465, 71

Tombesi, F., Cappi, M., Reeves, J. N., et al. 2010, A\&A, 521, A57

Tombesi, F., Cappi, M., Reeves, J. N., et al. 2013, MNRAS, 430, 1102

Tombesi, F., Meléndez, M., Veilleux, S., et al. 2015, Natur, 519, 436

Tremaine, S., Gebhardt, K., Bender, R., et al. 2002, ApJ, 574, 740

Tripathi, S., Misra, R., Dewangan, G., \& Rastogi, S. 2011, ApJL, 736, L37

Turner, A. K., Fabian, A. C., Lee, J. C., \& Vaughan, S. 2004, MNRAS, 353,319

Turner, T. J., Reeves, J. N., Kraemer, S. B., \& Miller, L. 2008, A\&A, 483, 161

Walton, D. J., Nardini, E., Fabian, A. C., Gallo, L. C., \& Reis, R. C. 2013, MNRAS, 428, 2901

Weisskopf, M. C., Hester, J. J., Tennant, A. F., et al. 2000, ApJL, 536, L81

Willingale, R., Starling, R. L. C., Beardmore, A. P., Tanvir, N. R., \& O'Brien, P. T. 2013, MNRAS, 431, 394

Wilms, J., Allen, A., \& McCray, R. 2000, ApJ, 542, 914

Yaqoob, T., McKernan, B., Kraemer, S. B., et al. 2003, ApJ, 582, 105

Zhang, S. N., Ji, L., Marshall, H. L., et al. 2011, MNRAS, 410, 2274

Zhou, X.-L., Zhang, S.-N., Wang, D.-X., \& Zhu, L. 2010, ApJ, 710, 16 